\title{
Geochemistry and Mantle Potential Temperature of Late Cenozoic Basaltic Rocks from the Northern and Western Continental Margins of the South China Sea
}

\author{
Xiaobo Yuan ${ }^{1,}$, Wang Ding ${ }^{2}$ \\ ${ }^{1}$ Institute of Marine Science, Hebei Normal University of Science and Technology, Qinhuangdao, China \\ ${ }^{2}$ Graduate School of Life and Environmental Sciences, University of Tsukuba, Tsukuba, Japan
}

Email address:

yuanxiaobo2011@163.com(Xiaobo Yuan), dingwang@geol.tsukuba.ac.jp (Wang Ding)

${ }^{*}$ Corresponding author

To cite this article:

Xiaobo Yuan, Wang Ding. Geochemistry and Mantle Potential Temperature of Late Cenozoic Basaltic Rocks from the Northern and Western Continental Margins of the South China Sea. Earth Sciences. Vol. 9, No. 5, 2020, pp. 178-191. doi: 10.11648/j.earth.20200905.14

Received: August 9, 2020; Accepted: September 10, 2020; Published: September 21, 2020

\begin{abstract}
Deep dynamic processes of the evolution of the South China Sea (SCS) are recorded by the large-scale magmatism in the SCS and its surrounding regions. The geochemical analysis of whole-rock and olivine phenocryst was conducted on the Late Cenozoic basaltic rocks, which are exposed in the continental margins of the northern (the Leiqiong Area) and western (the Kontum Area) SCS. Consistent geochemical characteristics were shown on the basaltic rocks based on the results, which are similar to OIB from intra-plate environment. Mantle potential temperature $\left(\mathrm{T}_{p}\right)$ was measured by $\mathrm{MgO}$ of parental magma compositions and by olivine-liquid equilibria thermometry, with an average of $1489^{\circ} \mathrm{C}$ beneath the Leizhou Peninsula, $1555^{\circ} \mathrm{C}$ beneath the Northern Hainan and $1458^{\circ} \mathrm{C}$ beneath the Kontum Area. Compared with $\mathrm{T}_{p}$ of SCS MORB, the mantle excess temperature is about $78-175^{\circ} \mathrm{C}$ in the studied areas. The highest excess temperature within typical plume-affected regions. Our results provide the evidence based on temperature for the existence of the Hainan Plume. The data of highest $\mathrm{T}_{p}$ under the North Hainan may indicate the upper reaches of mantle plume. The mantle source of post-spreading magmatism in SCS and its surrounding region is likely to be correlated to that of pre-spreading volcanic activities in the northern continental margins.
\end{abstract}

Keywords: Hainan Plume, Mantle Potential Temperature, Geochemistry, The South China Sea, Post-Spreading

\section{Introduction}

The Cenozoic basaltic rocks are widely covered in the South China Sea (SCS) and its surrounding region. In relation to the evolution of the SCS [1-2], the temporal and spatial distribution of Cenozoic magmatism in the areas can be divided into three stages [3-4]. Firstly the pre-spreading stage ( $>\sim 32 \mathrm{Ma})$, when scattered around the northern continental margin, magmatism erupted bimodal rift volcanic rocks [5-7]; Secondly the SCS basin spreading $(\sim 32-\sim 15.5 \mathrm{Ma})$, magmatism concentrated in the ocean basin, produced mainly middle-ocean ridge basalts (MORBs) [8-9]; Thirdly the post-spreading stage $(<\sim 15 \mathrm{Ma})$, intraplate magmatism expanded largely into the Leiqiong and adjacent areas in the South China, the Indochina Peninsula, and the SCS basin itself (Figure 1). Such temporal-spatial changes of magmatism have undoubtedly reveal the important signs about the evolution of the SCS and related deep dynamics.
There are several studies focusing on the post-spreading basaltic rocks from continental margins of the northern and western SCS [10-20]. Ocean Island Basalt-like (OIB-like) geochemical characteristics were shown on these basaltic rocks, originated from the mixture of the Depleted MORB Mantle (DMM) with Enriched Mantle 2 (EM-2) [20-21]. However, there are some debates about the origin of EM-2 like source. Some researchers considered continental lithospheric mantle [13-14], while others suggested a deep mantle plume source [1920, 22]. Therefore, the question about the existence of mantle plume becomes more important. In recent years, the Hainan Plume underlying the SCS region was proposed based on geophysical investigation [23-26]. A low-velocity column beneath the northern part of Hainan Island has extended from the shallow depths down to mantle transition zone [24]. Although geophysical studies suggested the existence of the Hainan Plume, the features of magmatism in this region are still slightly different 
from that in typical plume-affected regions. More evidence should be presented for the existence of Hainan plume.
Furthermore, the detail of thermal structure of the Hainan Plume and its effect on post-spreading magmatism are still unclear.

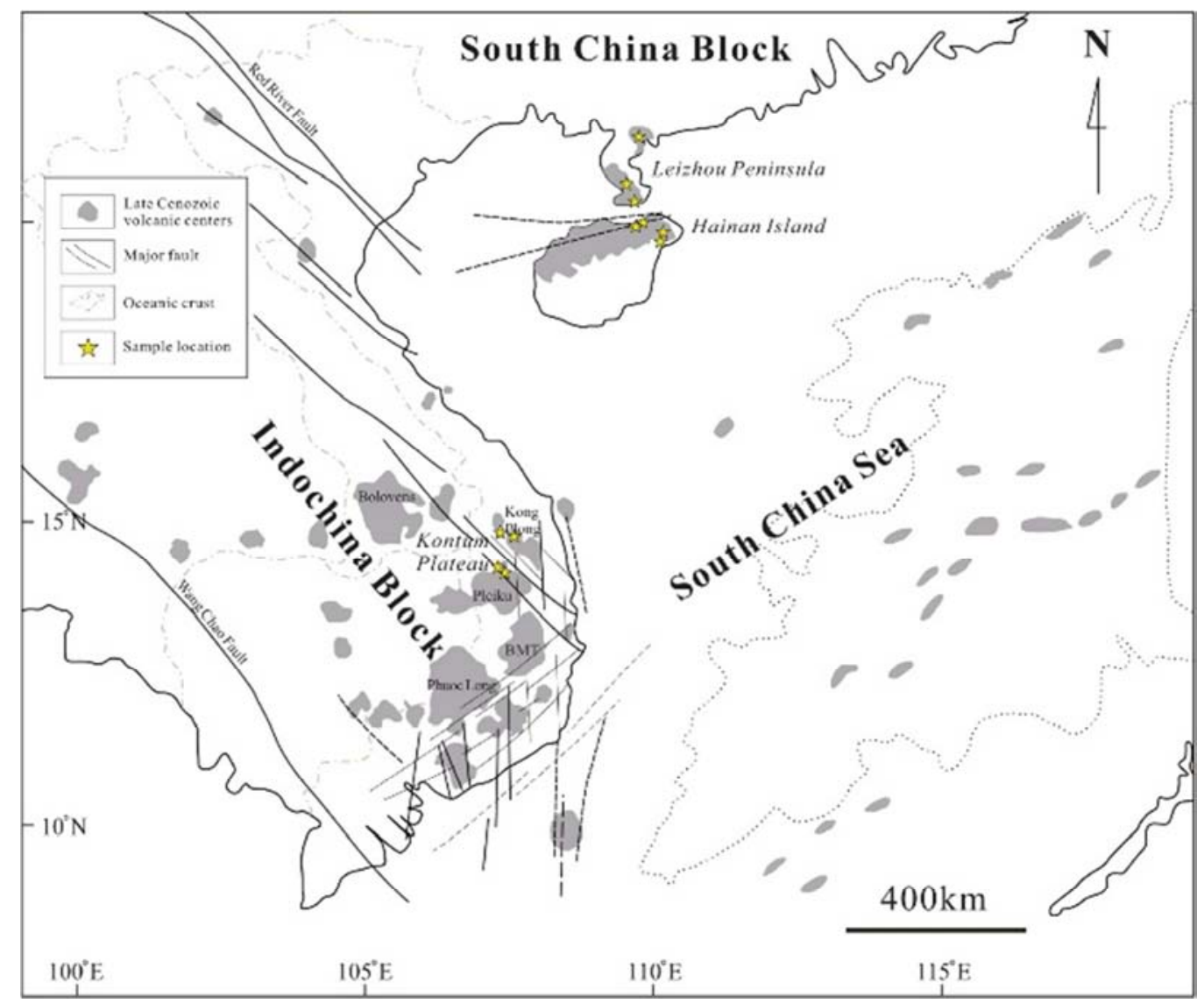

Figure 1. Map of South China Sea and its surrounding region showing SCS basin (dot line), fault systems (thin and dash line), Late Cenozoic basaltic volcanic centers (shaded) and sampling locations (yellow star) (after [13]).

The mantle potential temperature $\left(\mathrm{T}_{p}\right)$ was used to distinguish between the mantle plume-related thermal anomalies and ambient mantle [27-31]. Considering the wide distribution of basaltic rocks in Late Cenozoic times, the data of $\mathrm{T}_{p}$ in different locations is helpful for a better understanding of the thermal structure of mantle plume.

In this study, we collected the Late Cenozoic basaltic rocks from the Leiqiong Area in South China and the Kontum Area in Central Vietnam to carry out a geochemical analysis on whole-rock and olivine composition for the estimation of the $\mathrm{T}_{p}$. Based on the results, we constrain the mantle plume structure and its possible influence on the evolution of the SCS.

\section{Geological Background and Sampling}

In the continental margin of northern SCS, basaltic rocks covered mainly in the northern part of Hainan Island and the adjacent Leizhou Peninsula (Leiqiong Area), being over 4000 $\mathrm{km}^{2}$ in areas and up to over $100 \mathrm{~m}$ in thickness [10]. Volcanic activities during the Late Cenozoic times in Leiqiong Area were divided into two stages [10]: The early stage (late Tertiary, $>3.5 \mathrm{Ma}$ ) dominated by flood type fissure eruption of quartz- or olivine-tholeiites, and the later phase (Quaternary, $<2 \mathrm{Ma}$ ) characterized by central type eruption of alkali olivine basalts and olivine tholeiites. Three samples from Leizhou
Peninsula were collected respectively from Huguangyan area, Yingfengling Mountain, and at Xuwen town with an eruptive period no more than 0.1Ma. Eight samples were obtained in northern part of Hainan Island: three from Maanling Mountain and Jinniuling Mountain in Haikou city, the eruption period no more than $0.1 \mathrm{Ma}$; five from Penglai Town, erupted at about 3.57-4.80 Ma (Figure 1) (Table 1).

Occurred in the Indochina Peninsula, the Late Cenozoic magmatism formed scattered volcanic centers (Figure 1). We collected samples from the Kong Plong and Pleiku volcanic centers around the Kontum Area in Central Vietnam (Figure 1) (Table 1). The Kong Plong volcanic center erupted during 10.5-16.5 Ma and 7.0-8.2 Ma, the basaltic rocks are interbedded with sandstone, siltstone, and claystone [32]. The magmatism in the Pleiku were later than in the Kong Plong, which preserved two eruptive episodes: the early episodes (6.5-3.4 Ma) dominated by quartz- or olivine- tholeiite and the later episodes (2.4-0.2 Ma) erupted olivine tholeiite, alkali basalt, and basanite [17]. Therefore, the ages of samples from Kong Plong and Pleiku are in the range between 16.5-7.0 Ma and 6.5-0.2 Ma, respectively.

All samples are fresh without any significant alterations. Porphyritic textures and vesicular structure were displayed in different rocks. The phenocrysts are mainly olivine, clinopyroxene and plagioclase. Plagioclase crystals are 
euhedral-subeuhedral and the display of polysynthetic twinning. The matrix shows intersertal texture consisting of volcanic glass and very tiny microcrystals of plagioclase, clinopyroxene.

\section{Analytical Methods}

The samples were worn off and ground to 200 mesh for whole-rock geochemical analyses.

The analysis on whole rock for its major and trace elements were performed at the Regional Geology and Minerals Survey of Hebei Province, Langfang, Hebei Province. The analysis of major elements was conducted by X-ray fluorescence (XRF) (RIKAGU RIX 2100), with standard GBW07105 and BCR-2 were used to monitor drift during measurements, and its analytical precision is $5 \%$ better. The analysis of trace elements, including rare earth elements (REE), were carried out by an inductively coupled plasma mass spectrometry (ICP-MS) (Agilent 7500a) and monitored by the GBW07105 (GSR-3). The accuracy of ICP-MS for trace elements is $5-10 \%$ better depending on the concentration of the given element. The experimental procedures can refer to [7].

Six samples (three Leiqiong basaltic rocks and three Kontum basaltic rocks) were one-side polished to make thin sections for microscopic observation and electron probe micro analyses (EPMA). Analyses on the major element analyses of olivine were carried out by using SHIMADZU EPMA-1720 instrument at the Experimental Center of China University of Geosciences, Beijing. Operating conditions were as follows: 15 $\mathrm{kV}$ accelerating voltage, $10 \mathrm{nA}$ beam current, and a beam spot diameter of $1 \mu \mathrm{m}$. Counting times were $50 \mathrm{~s}$ for all elements. The data were regressed by oxide-ZAF correction methods.

\section{Results}

\subsection{Major and Trace Element Compositions}

Results of whole-rock major elements and trace elements of samples from the Leiqiong and Kontum Areas are listed in Table 1.

The $\mathrm{SiO}_{2}$ contents of the Leiqiong basaltic rocks range from $48.86-54.39 \%$ with an average of $51.10 \%$; samples from the Kontum Area show slightly lower $\mathrm{SiO}_{2}$ contents varying

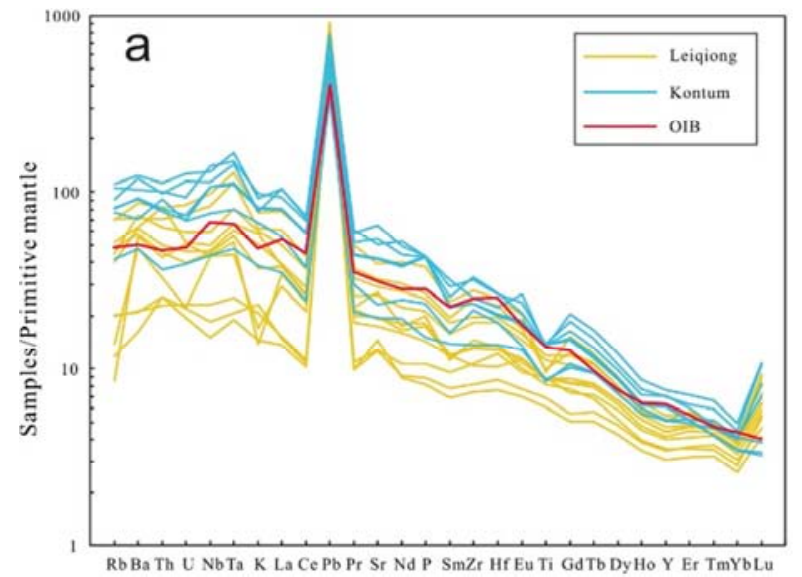

$44.31-50.76 \%$ (average 46.58\%). Total alkalis $\left(\mathrm{Na}_{2} \mathrm{O}+\mathrm{K}_{2} \mathrm{O}\right)$ are higher than $3.1 \%$ for all samples. In a total alkalis-silica (TAS) diagram (Figure 2), samples from the Leiqiong Area are classified as basalt, basaltic andesite, trachybasalt, and basaltic trachyandesite, varying from alkaline to calc-alkaline series, while samples from the Kontum Area belong to the alkaline series with one exception (VN024-1). The Mg\# values $\left(\mathrm{Mg} \#=100 * \mathrm{Mg} /\left(\mathrm{Mg}+\mathrm{Fe}^{2+}\right)\right.$ of Leiqiong basaltic rocks show the ranges of 53.40-63.15 (average 57.29), similar to the range of Kontum basaltic rocks (47.92-60.17, average 57.54).

In the distribution pattern of primitive mantle-normalized trace element (Figure 3a), a typical OIB-like pattern is shown in all samples, which are featured by enriched in large ion lithophile elements (LILE) and high field strength elements (HFSE), without negative $\mathrm{Pb}$ anomalies. The $\mathrm{Ni}, \mathrm{Co}, \mathrm{Cr}$ of samples from Leiqiong Area are 86.9-361.5 ppm, 39.2-46.9 ppm, and 234-472.2 ppm, respectively. Those of rocks from Kontum Plateau are 167-252 ppm, 50.7-69.6 ppm and 234$287 \mathrm{ppm}$, respectively. The $\mathrm{Mg \#}$ values and the $\mathrm{Ni}$ and $\mathrm{Cr}$ contents of all samples are far lower than those for partial melts from peridotite mantle $(\mathrm{Mg} \#>70, \mathrm{Ni}>400-500 \mathrm{ppm}$, Cr>1000 ppm; [35-36]).

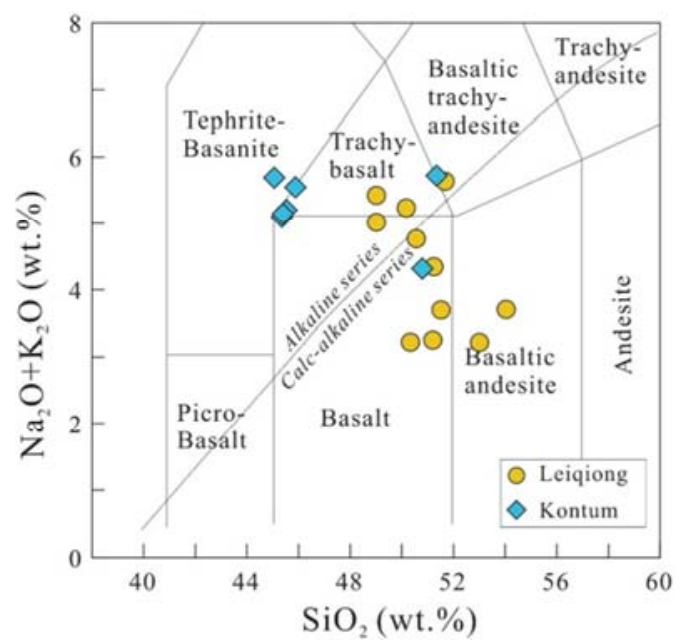

Figure 2. A rock classification diagram of the basaltic rocks from this study, according to their $\mathrm{Na}_{2} \mathrm{O}+\mathrm{K}_{2} \mathrm{O}$ and $\mathrm{SiO}_{2}$ contents. The dashed line separating alkaline and sub-alkaline basalts is from [33].

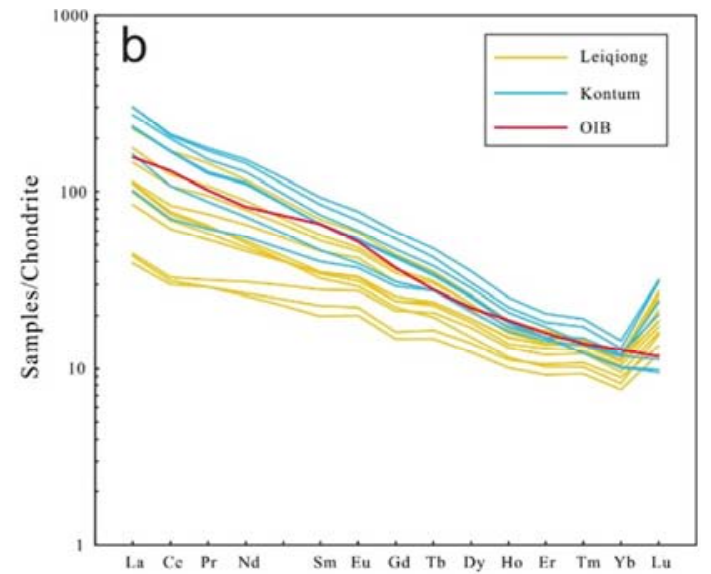

Figure 3. (a) Primitive mantle-normalized spider grams for basaltic rocks from Leiqiong Area and Kontum Plateau. Data for the primitive mantle and OIBs are from [34]. (b) Chondrite-normalized spider grams for basaltic rocks from Leiqiong Area and Kontum Plateau. Data for the chondrite and OIBs are from [34]. 
In the Leiqiong basaltic rocks, total REE contents ( $\Sigma$ REE) are shown in a range of 59.38-263.33 ppm, while the Kontum basaltic rocks display larger range of $126.55-338.88 \mathrm{ppm}$. From the chondrite-normalized REE diagram (Figure 3b), a light rare earth element (LREE) enriched pattern is shown in all samples. The LREE/HREE are 3.71-10.50 for the Leiqiong basaltic rocks and 5.61-10.99 for the Kontum basaltic rocks, indicating the high degree of fractionation between LREE and HREE. The Leiqiong basaltic rocks show a slightly positive anomaly of $\mathrm{Eu}(\delta \mathrm{Eu}=1.05-1.16)$, while no $\mathrm{Eu}$ anomaly $(\delta \mathrm{Eu}=0.98-1.07)$ in the Kontum basaltic rocks (Figure 3b). Both rocks, however, show enrichment of $(\mathrm{La} / \mathrm{Yb})_{N}(4.44-18.99$ and 8.47-23.31, respectively).

\subsection{Mineral Chemical Characteristics}

Table 2 listed the results of the analyses on electron probe micro of the olivine from the Leiqiong and Kontum basaltic rocks. For all measured olivines, $\mathrm{Ni}$ and $\mathrm{Al}$ are ranged from $6717 * 10^{-6}-8570^{*} 10^{-6}$ and $229 * 10^{-6}-1096^{*} 10^{-6}$, respectively, within the contents range of olivine phenocrysts $\left(\mathrm{Ni}<2200 * 10^{-6}\right.$ or $>3400 * 10^{-6}, \mathrm{Al}>130^{*} 10^{-6} ;$; [37]). The $\mathrm{CaO}$ contents are from 0.11 to $0.34 \%$, which are much higher than mantle peridotite xenoliths $(\mathrm{CaO}<0.1 \%$; [38]). As a result, the measured olivines are phenocrysts.

The olivine phenocrysts show slight normal zonation with its Fo values decrease from core to crust. The Fo values of olivine phenocrysts from the Leiqiong Area are ranged from 71.96 to 90.88 , while that of the Kontum Area range from 62.53 to 89.27 . The $\mathrm{NiO}$ contents decrease with the decreasing of Fo values, most of olivines are comparable with Hainan basalt olivines (Figure 4).

Table 1. Whole-rock major elements and trace elements of basaltic rocks from the Leiqiong and Kontum Areas.

\begin{tabular}{|c|c|c|c|c|c|c|c|c|c|c|c|}
\hline \multirow{2}{*}{$\begin{array}{l}\text { Area } \\
\text { Location }\end{array}$} & \multicolumn{11}{|l|}{ Leiqiong Area } \\
\hline & \multicolumn{3}{|c|}{ Leizhou Peninsula } & \multicolumn{8}{|c|}{ Northern Hainan } \\
\hline GPS & $\begin{array}{l}\mathrm{N} \\
21^{\circ} 08^{\prime} 30.43^{\prime \prime} \\
\end{array}$ & $\begin{array}{l}\mathrm{N} \\
20^{\circ} 34^{\prime} 51.10^{\prime \prime} \\
\end{array}$ & $\begin{array}{l}\mathrm{N} \\
20^{\circ} 16^{\prime} 38.56^{\prime \prime} \\
\end{array}$ & $\begin{array}{l}\mathrm{N} \\
19^{\circ} 55^{\prime} 50.48^{\prime \prime} \\
\end{array}$ & $\begin{array}{l}\mathrm{N} \\
19^{\circ} 55^{\prime} 50.48^{\prime \prime} \\
\end{array}$ & $\begin{array}{l}\mathrm{N} \\
20^{\circ} 00^{\prime} 28.75^{\prime \prime}\end{array}$ & $\begin{array}{l}\mathrm{N} \\
19^{\circ} 30^{\prime} 11.66^{\prime \prime}\end{array}$ & $\begin{array}{l}\mathrm{N} \\
19^{\circ} 30^{\prime} 11.66^{\prime \prime} \\
\end{array}$ & $\begin{array}{l}\mathrm{N} \\
19^{\circ} 34^{\prime} 41.24^{\prime \prime} \\
\end{array}$ & $\begin{array}{l}\mathrm{N} \\
19^{\circ} 34^{\prime} 41.24^{\prime \prime} \\
\end{array}$ & $\begin{array}{l}\mathrm{N} \\
19^{\circ} 34^{\prime} 41.24^{\prime \prime} \\
\end{array}$ \\
\hline Position & $\begin{array}{l}E \\
110^{\circ} 16^{\prime} 13.35^{\prime \prime} \\
\end{array}$ & $\begin{array}{l}\mathrm{E} \\
110^{\circ} 10^{\prime} 29.78^{\prime \prime}\end{array}$ & $\begin{array}{l}\mathrm{E} \\
110^{\circ} 15^{\prime} 09.07^{\prime \prime}\end{array}$ & $\begin{array}{l}\mathrm{E} \\
110^{\circ} 13^{\prime} 41.83^{\prime \prime}\end{array}$ & $\begin{array}{l}\mathrm{E} \\
110^{\circ} 13^{\prime} 41.83^{\prime \prime}\end{array}$ & $\begin{array}{l}\mathrm{E} \\
110^{\circ} 18^{\prime} 51.93^{\prime \prime}\end{array}$ & $\begin{array}{l}\mathrm{E} \\
110^{\circ} 32^{\prime} 01.69^{\prime \prime}\end{array}$ & $\begin{array}{l}\mathrm{E} \\
110^{\circ} 32^{\prime} 01.69^{\prime \prime}\end{array}$ & $\begin{array}{l}\mathrm{E} \\
110^{\circ} 38^{\prime 38.04 \prime \prime}\end{array}$ & $\begin{array}{l}\mathrm{E} \\
110^{\circ} 38^{\prime 38.04 \prime \prime}\end{array}$ & $\begin{array}{l}\mathrm{E} \\
110^{\circ} 38^{\prime 38.04 \prime \prime}\end{array}$ \\
\hline Sample No. & MM28-1 & MM30-2 & MM32-1 & H001-1 & HK01-2 & HK02-2 & HK05-2 & HK05-7 & HK06-4 & HK06-6 & HK06-8 \\
\hline \multicolumn{12}{|c|}{ Major Oxides (wt.\%) } \\
\hline $\mathrm{SiO}_{2}$ & 50.86 & 53.16 & 51.89 & 50.95 & 49.19 & 54.39 & 51.68 & 48.86 & 51.07 & 50.69 & 49.42 \\
\hline $\mathrm{Al}_{2} \mathrm{O}_{3}$ & 14.9 & 14.57 & 14.86 & 14.23 & 13.78 & 13.84 & 13.12 & 12.46 & 13.4 & 13.74 & 13.89 \\
\hline $\mathrm{TiO}_{2}$ & 1.77 & 1.49 & 1.32 & 2.44 & 2.59 & 1.94 & 2.16 & 2.08 & 1.94 & 1.92 & 1.92 \\
\hline $\mathrm{Fe}_{2} \mathrm{O}_{3}$ & 2.26 & 3.48 & 2.06 & 4.26 & 10.23 & 1.97 & 3.93 & 3.18 & 3.99 & 5 & 5.54 \\
\hline $\mathrm{FeO}$ & 7.22 & 6.58 & 7.9 & 6.36 & 1.11 & 8.06 & 7.1 & 7.98 & 6.86 & 5.49 & 5.66 \\
\hline $\mathrm{CaO}$ & 8.28 & 8.57 & 8.6 & 8.83 & 9.05 & 8.2 & 6.71 & 6.58 & 8.31 & 9.02 & 8.53 \\
\hline $\mathrm{MgO}$ & 8.36 & 7.23 & 8.3 & 6.55 & 6.92 & 6.5 & 7.37 & 10.42 & 7.63 & 7.55 & 7.88 \\
\hline $\mathrm{K}_{2} \mathrm{O}$ & 1.74 & 0.44 & 0.7 & 1.53 & 1.81 & 0.62 & 1.88 & 2.31 & 1.11 & 0.42 & 0.51 \\
\hline $\mathrm{Na}_{2} \mathrm{O}$ & 3.49 & 2.78 & 2.99 & 3.19 & 3.16 & 3.06 & 3.69 & 3.03 & 3.16 & 2.76 & 2.62 \\
\hline $\mathrm{MnO}$ & 0.14 & 0.14 & 0.15 & 0.15 & 0.15 & 0.14 & 0.15 & 0.17 & 0.15 & 0.15 & 0.16 \\
\hline $\mathrm{P}_{2} \mathrm{O}_{5}$ & 0.43 & 0.19 & 0.18 & 0.46 & 0.54 & 0.23 & 0.59 & 0.83 & 0.39 & 0.38 & 0.31 \\
\hline LOI & 0.32 & 1.2 & 0.89 & 0.8 & 1.2 & 0.86 & 1.34 & 1.74 & 1.77 & 2.59 & 3.33 \\
\hline Total & 99.77 & 99.83 & 99.83 & 99.75 & 99.73 & 99.82 & 99.71 & 99.64 & 99.78 & 99.78 & 99.8 \\
\hline Mg\# & 61.69 & 57.03 & 60.25 & 53.4 & 54.46 & 54.08 & 55.26 & 63.15 & 56.54 & 57.41 & 56.88 \\
\hline \multicolumn{12}{|c|}{ Trace elements (ppm) } \\
\hline Y & 16.14 & 15.77 & 13.9 & 21.38 & 23.19 & 18.16 & 23.14 & 24.74 & 20.02 & 18.98 & 20.23 \\
\hline $\mathrm{La}$ & 26.26 & 9.33 & 10.19 & 26.9 & 34.52 & 12.53 & 41.84 & 53.75 & 25.91 & 23.64 & 20.1 \\
\hline $\mathrm{Ce}$ & 47.05 & 48.23 & 39.2 & 51.03 & 65.64 & 29.97 & 76.48 & 103.55 & 45.56 & 42.27 & 37.62 \\
\hline $\operatorname{Pr}$ & 6.13 & 2.73 & 2.75 & 7.11 & 9 & 3.01 & 10.16 & 13.73 & 5.99 & 5.53 & 5.09 \\
\hline $\mathrm{Nd}$ & 23.74 & 12.37 & 12.01 & 30.32 & 36.94 & 14.47 & 41.08 & 54.46 & 24.61 & 22.55 & 21.62 \\
\hline $\mathrm{Sm}$ & 4.98 & 3.46 & 3.04 & 7.05 & 8.09 & 4.25 & 8.63 & 10.61 & 5.35 & 5.22 & 5.29 \\
\hline $\mathrm{Eu}$ & 1.7 & 1.29 & 1.16 & 2.44 & 2.67 & 1.6 & 2.81 & 3.39 & 1.92 & 1.81 & 1.9 \\
\hline Gd & 4.56 & 3.32 & 3.02 & 6.32 & 7.12 & 4.34 & 7.42 & 9.08 & 5.22 & 4.94 & 4.96 \\
\hline $\mathrm{Tb}$ & 0.73 & 0.61 & 0.55 & 1.03 & 1.13 & 0.77 & 1.17 & 1.33 & 0.89 & 0.85 & 0.88 \\
\hline Dy & 3.84 & 3.54 & 3.13 & 5.44 & 5.89 & 4.31 & 5.88 & 6.59 & 4.86 & 4.62 & 4.86 \\
\hline Ho & 0.65 & 0.63 & 0.57 & 0.9 & 0.97 & 0.74 & 0.95 & 1.06 & 0.85 & 0.78 & 0.85 \\
\hline $\mathrm{Er}$ & 1.69 & 1.74 & 1.52 & 2.31 & 2.74 & 1.98 & 2.47 & 2.69 & 2.28 & 2.15 & 2.25 \\
\hline $\mathrm{Tm}$ & 0.26 & 0.28 & 0.24 & 0.35 & 0.37 & 0.31 & 0.37 & 0.36 & 0.34 & 0.32 & 0.34 \\
\hline $\mathrm{Yb}$ & 1.4 & 1.51 & 1.29 & 1.85 & 1.98 & 1.62 & 1.87 & 2.03 & 1.88 & 1.77 & 1.9 \\
\hline $\mathrm{Lu}$ & 0.39 & 0.34 & 0.31 & 0.54 & 0.62 & 0.41 & 0.66 & 0.69 & 0.47 & 0.44 & 0.48 \\
\hline $\mathrm{Li}$ & 7.28 & 5.27 & 4.07 & 6.05 & 8.46 & 4.59 & 5.52 & 6.9 & 5.21 & 4.04 & 6.53 \\
\hline $\mathrm{Be}$ & 1.53 & 0.7 & 0.72 & 1.41 & 1.55 & 0.85 & 1.95 & 2.11 & 0.93 & 1.02 & 0.94 \\
\hline $\mathrm{Sc}$ & 14.23 & 15.66 & 15.65 & 16.28 & 15.89 & 15.82 & 12.88 & 11.14 & 16.69 & 16.22 & 16.42 \\
\hline V & 149.69 & 138.1 & 137.97 & 262.74 & 239.6 & 138.85 & 126.63 & 113.97 & 147.17 & 209.67 & 221.15 \\
\hline $\mathrm{Cr}$ & 244.37 & 305.78 & 271.61 & 293.12 & 296.7 & 265.23 & 331.46 & 472.21 & 234 & 254.57 & 253.11 \\
\hline $\mathrm{Co}$ & 42.62 & 41.62 & 46.86 & 39.18 & 40.18 & 39.53 & 41.83 & 45.27 & 42.61 & 41.86 & 44.93 \\
\hline $\mathrm{Ni}$ & 176.27 & 150.18 & 189.37 & 86.95 & 99.18 & 146.63 & 227.44 & 361.53 & 168.39 & 161.28 & 175.71 \\
\hline
\end{tabular}




\begin{tabular}{|c|c|c|c|c|c|c|c|c|c|c|c|}
\hline \multirow{2}{*}{$\begin{array}{l}\text { Area } \\
\text { Location }\end{array}$} & \multicolumn{11}{|l|}{ Leiqiong Area } \\
\hline & \multicolumn{3}{|c|}{ Leizhou Peninsula } & \multicolumn{8}{|c|}{ Northern Hainan } \\
\hline GPS & $\begin{array}{l}\mathrm{N} \\
21^{\circ} 08^{\prime} 30.43^{\prime \prime} \\
\end{array}$ & $\begin{array}{l}\mathrm{N} \\
20^{\circ} 34^{\prime} 51.10^{\prime \prime}\end{array}$ & $\begin{array}{l}\mathrm{N} \\
20^{\circ} 16^{\prime} 38.56^{\prime \prime} \\
\end{array}$ & $\begin{array}{l}\mathrm{N} \\
19^{\circ} 55^{\prime} 50.48^{\prime \prime}\end{array}$ & $\begin{array}{l}\mathrm{N} \\
19^{\circ} 55^{\prime} 50.48^{\prime \prime}\end{array}$ & $\begin{array}{l}\mathrm{N} \\
20^{\circ} 00^{\prime} 28.75^{\prime \prime} \\
\end{array}$ & $\begin{array}{l}\mathrm{N} \\
19^{\circ} 30^{\prime} 11.66^{\prime \prime} \\
\end{array}$ & $\begin{array}{l}\mathrm{N} \\
19^{\circ} 30^{\prime} 11.66^{\prime \prime}\end{array}$ & $\begin{array}{l}\mathrm{N} \\
19^{\circ} 34^{\prime} 41.24^{\prime \prime}\end{array}$ & $\begin{array}{l}\mathrm{N} \\
19^{\circ} 34^{\prime} 41.24^{\prime \prime}\end{array}$ & $\begin{array}{l}\mathrm{N} \\
19^{\circ} 34^{\prime} 41.24^{\prime \prime}\end{array}$ \\
\hline Position & $\begin{array}{l}\mathrm{E} \\
110^{\circ} 16^{\prime} 13.35^{\prime \prime}\end{array}$ & $\begin{array}{l}\mathrm{E} \\
110^{\circ} 10^{\prime} 29.78^{\prime \prime}\end{array}$ & $\begin{array}{l}E \\
110^{\circ} 15^{\prime} 09.07^{\prime \prime}\end{array}$ & $\begin{array}{l}\mathrm{E} \\
110^{\circ} 13^{\prime} 41.83^{\prime \prime}\end{array}$ & $\begin{array}{l}\mathrm{E} \\
110^{\circ} 13^{\prime} 41.83^{\prime \prime}\end{array}$ & $\begin{array}{l}E \\
110^{\circ} 18^{\prime} 51.93^{\prime \prime}\end{array}$ & $\begin{array}{l}\mathrm{E} \\
110^{\circ} 32^{\prime} 01.69^{\prime \prime}\end{array}$ & $\begin{array}{l}\mathrm{E} \\
110^{\circ} 32^{\prime} 01.69^{\prime \prime}\end{array}$ & $\begin{array}{l}\mathrm{E} \\
110^{\circ} 38^{\prime} 38.04^{\prime \prime}\end{array}$ & $\begin{array}{l}\mathrm{E} \\
110^{\circ} 38^{\prime} 38.04^{\prime \prime}\end{array}$ & $\begin{array}{l}\mathrm{E} \\
110^{\circ} 38^{\prime} 38.04^{\prime \prime}\end{array}$ \\
\hline Sample No. & MM28-1 & MM30-2 & MM32-1 & H001-1 & HK01-2 & HK02-2 & HK05-2 & HK05-7 & HK06-4 & HK06-6 & HK06-8 \\
\hline $\mathrm{Cu}$ & 54.35 & 50.39 & 51.06 & 43.79 & 58.47 & 57.55 & 44.14 & 35.98 & 52.12 & 51.71 & 51.51 \\
\hline $\mathrm{Ga}$ & 18.91 & 16.88 & 16.95 & 21.68 & 21.02 & 19.08 & 22.51 & 20.75 & 19.3 & 19 & 19.23 \\
\hline $\mathrm{Rb}$ & 33.11 & 7.51 & 12.8 & 25.56 & 28.62 & 12.68 & 44.76 & 44.1 & 30.74 & 5.43 & 8.76 \\
\hline $\mathrm{Sr}$ & 575.5 & 266.2 & 308 & 554.4 & 614.1 & 271 & 675.6 & 828.5 & 404.1 & 418.1 & 366 \\
\hline $\mathrm{Zr}$ & 163.08 & 91.12 & 82.59 & 205.48 & 222.16 & 120.21 & 277.41 & 311.42 & 145.99 & 148.94 & 116.3 \\
\hline $\mathrm{Nb}$ & 35.61 & 10.7 & 13.19 & 33.19 & 42.46 & 16.57 & 59.72 & 69.64 & 32.54 & 31.01 & 30.87 \\
\hline Mo & 1.32 & 0.6 & 0.68 & 1.66 & 1.04 & 0.81 & 2.85 & 1.23 & 0.99 & 1.22 & 0.51 \\
\hline In & 0.05 & 0.05 & 0.04 & 0.05 & 0.07 & 0.06 & 0.07 & 0.07 & 0.06 & 0.06 & 0.06 \\
\hline $\mathrm{Cs}$ & 0.99 & 0.21 & 0.35 & 0.38 & 0.36 & 0.36 & 0.2 & 0.47 & 0.54 & 0.24 & 0.2 \\
\hline $\mathrm{Ba}$ & 439.4 & 113 & 146.4 & 436.3 & 518.4 & 147.6 & 498.2 & 604.4 & 404.7 & 390.6 & 335.7 \\
\hline Hf & 4.13 & 2.68 & 2.36 & 5.67 & 6 & 3.75 & 7.09 & 7.65 & 3.96 & 4.17 & 3.2 \\
\hline $\mathrm{Ta}$ & 2.73 & 0.78 & 0.85 & 2.49 & 3.31 & 1.04 & 4.66 & 5.3 & 2.32 & 2.14 & 1.81 \\
\hline W & 0.55 & 0.26 & 0.24 & 0.53 & 0.63 & 0.33 & 0.44 & 0.25 & 0.43 & 0.38 & 0.12 \\
\hline $\mathrm{Tl}$ & 0.12 & 0.07 & 0.07 & 0.06 & 0.03 & 0.06 & 0.05 & 0.08 & 0.05 & 0.02 & 0.03 \\
\hline $\mathrm{Pb}$ & 6.43 & 4.74 & 4.82 & 6.04 & 7.25 & 4.46 & 5.77 & 6.34 & 5.22 & 4.98 & 4.66 \\
\hline $\mathrm{Bi}$ & 0.02 & 0.02 & 0.03 & 0.02 & 0.03 & 0.03 & 0.02 & 0.03 & 0.01 & 0.02 & 0.02 \\
\hline $\mathrm{Th}$ & 7.01 & 2.16 & 2.16 & 4.24 & 5.36 & 1.94 & 6.02 & 6.98 & 3.86 & 3.62 & 2.75 \\
\hline $\mathrm{U}$ & 1.09 & 0.41 & 0.46 & 0.97 & 1.24 & 0.49 & 1.57 & 1.79 & 0.84 & 1.07 & 0.47 \\
\hline
\end{tabular}

Table 1. Continued.

\begin{tabular}{|c|c|c|c|c|c|c|c|}
\hline Area & Kontum Area & & & & & & \\
\hline Location & Kong Plong & Pleiku & & & & & \\
\hline \multirow{2}{*}{ GPS Position } & N 144' $16.07^{\prime \prime}$ & N 14'22'6.73" & N 14'22'6.73" & N 14'16'59.72" & N 14'16'45.99" & N 14'16'45.99" & N 14'16'45.99" \\
\hline & E 108 $27^{\circ} 43.33^{\prime \prime}$ & E $107^{\circ} 55^{\prime 36.93 "}$ & E $107^{\circ} 55 ' 36.93 "$ & E $107^{\circ} 57^{\prime} 20.31^{\prime \prime}$ & E $107^{\circ} 58^{\prime} 14.39^{\prime \prime}$ & E $107^{\circ} 58^{\prime} 14.39^{\prime \prime}$ & E $107^{\circ} 58^{\prime} 14.39^{\prime \prime}$ \\
\hline Sample No. & VN15-1 & VN23-1 & VN23-2 & VN24-1 & VN25-1 & VN25-2 & VN25-3 \\
\hline \multicolumn{8}{|c|}{ Major Oxides (wt.\%) } \\
\hline $\mathrm{SiO}_{2}$ & 50.24 & 44.31 & 45.32 & 50.76 & 45.24 & 44.94 & 45.27 \\
\hline $\mathrm{Al}_{2} \mathrm{O}_{3}$ & 15.32 & 12.39 & 12.68 & 13.56 & 12.43 & 12.37 & 12.55 \\
\hline $\mathrm{Fe}_{2} \mathrm{O}_{3}$ & 6.96 & 2.4 & 3.02 & 1.85 & 3.18 & 2.56 & 3.08 \\
\hline $\mathrm{FeO}$ & 3.81 & 9.53 & 8.69 & 9.21 & 9.1 & 9.6 & 9.12 \\
\hline $\mathrm{CaO}$ & 6.63 & 8.5 & 8.16 & 8.47 & 8.48 & 8.52 & 8.43 \\
\hline $\mathrm{MgO}$ & 5.2 & 9.78 & 9.6 & 7.66 & 9.93 & 10.09 & 9.82 \\
\hline $\mathrm{K}_{2} \mathrm{O}$ & 2.01 & 2.78 & 2.97 & 1.15 & 2.44 & 2.35 & 2.45 \\
\hline $\mathrm{Na}_{2} \mathrm{O}$ & 3.52 & 2.74 & 2.43 & 3.11 & 2.64 & 2.65 & 2.62 \\
\hline $\mathrm{MnO}$ & 0.12 & 0.17 & 0.18 & 0.17 & 0.18 & 0.17 & 0.18 \\
\hline LOI & 3.67 & 3.12 & 2.75 & 1.66 & 2.22 & 2.61 & 2.28 \\
\hline Total & 99.81 & 99.66 & 99.68 & 99.8 & 99.73 & 99.7 & 99.71 \\
\hline $\mathrm{Mg \#}$ & 47.92 & 59.85 & 59.99 & 55.67 & 59.67 & 60.17 & 59.53 \\
\hline \multicolumn{8}{|c|}{ Trace elements (ppm) } \\
\hline $\mathrm{Y}$ & 23.28 & 31.53 & 32.18 & 23.72 & 27.62 & 34.62 & 27.85 \\
\hline $\mathrm{La}$ & 38.27 & 71.58 & 64.33 & 24.19 & 54.55 & 71.45 & 55.16 \\
\hline $\mathrm{Ce}$ & 65.82 & 126.27 & 121.95 & 43.22 & 103.18 & 129.51 & 104.33 \\
\hline $\operatorname{Pr}$ & 8.27 & 16.17 & 14.31 & 5.81 & 12.01 & 16.74 & 12.23 \\
\hline $\mathrm{Nd}$ & 33.45 & 67.2 & 59.94 & 25.9 & 51.35 & 71.03 & 52.25 \\
\hline $\mathrm{Sm}$ & 7.12 & 12.93 & 11.31 & 6.13 & 9.92 & 14.15 & 10.07 \\
\hline $\mathrm{Eu}$ & 2.27 & 3.99 & 3.48 & 2.15 & 3.05 & 4.47 & 3.15 \\
\hline $\mathrm{Gd}$ & 6.37 & 11.06 & 9.74 & 6.03 & 8.68 & 12.22 & 8.85 \\
\hline $\mathrm{Tb}$ & 1.03 & 1.6 & 1.41 & 1.03 & 1.25 & 1.79 & 1.28 \\
\hline Dy & 5.34 & 7.8 & 7.21 & 5.69 & 6.41 & 8.97 & 6.53 \\
\hline Но & 0.92 & 1.25 & 1.17 & 0.98 & 1.02 & 1.42 & 1.04 \\
\hline $\mathrm{Er}$ & 2.38 & 3.04 & 2.87 & 2.49 & 2.47 & 3.4 & 2.5 \\
\hline $\mathrm{Lu}$ & 0.61 & 0.78 & 0.29 & 0.51 & 0.24 & 0.8 & 0.25 \\
\hline $\mathrm{Li}$ & 8.34 & 6.98 & 4.82 & 4.66 & 6.38 & 7.1 & 6.37 \\
\hline $\mathrm{Be}$ & 1.59 & 2.61 & 2.79 & 1.14 & 2.16 & 2.04 & 2.17 \\
\hline
\end{tabular}




\begin{tabular}{|c|c|c|c|c|c|c|c|}
\hline Area & Kontum Area & & & & & & \\
\hline Location & Kong Plong & Pleiku & & & & & \\
\hline \multirow{2}{*}{ GPS Position } & N 14²'16.07" & N 14²2'6.73" & N 14'22'6.73" & N 14¹6'59.72" & N 14'16'45.99" & N 14'16'45.99" & N 1416'45.99" \\
\hline & E 108'27'43.33" & E 107'55'36.93" & E 107'55'36.93" & E $107^{\circ} 57^{\prime 20.31 "}$ & E 107'58'14.39" & E 107'58'14.39" & E $107^{\circ} 58^{\prime} 14.39^{\prime \prime}$ \\
\hline Sample No. & VN15-1 & VN23-1 & VN23-2 & VN24-1 & VN25-1 & VN25-2 & VN25-3 \\
\hline $\mathrm{Sc}$ & 20.66 & 19.1 & 12.66 & 21.97 & 12.82 & 20.27 & 10.55 \\
\hline V & 145.44 & 179.52 & 173.37 & 147.04 & 177.07 & 187.12 & 177.81 \\
\hline $\mathrm{Cr}$ & 233.8 & 247.41 & 274.4 & 287.28 & 251.43 & 264.6 & 249.08 \\
\hline Co & 50.68 & 65.04 & 54.13 & 54.96 & 56.2 & 69.61 & 56.39 \\
\hline $\mathrm{Ni}$ & 167.2 & 231.44 & 228.21 & 201.41 & 226.6 & 252.45 & 228.46 \\
\hline $\mathrm{Cu}$ & 63.59 & 62.29 & 46.84 & 81.28 & 50.17 & 70.37 & 51.71 \\
\hline $\mathrm{Zn}$ & 114.77 & 152.72 & 166.6 & 114.54 & 156.08 & 154.1 & 157.21 \\
\hline $\mathrm{Ga}$ & 22.15 & 24.68 & 25.29 & 20.14 & 23.14 & 23.94 & 23.32 \\
\hline $\mathrm{Rb}$ & 48.8 & 70.13 & 66.72 & 26.36 & 51.61 & 58.14 & 51.23 \\
\hline $\mathrm{Sr}$ & 485.93 & 1372.5 & 1155.93 & 409.73 & 878.34 & 1062.75 & 887.97 \\
\hline $\mathrm{Zr}$ & 241.76 & 354.37 & 369.9 & 153.73 & 263.22 & 283.23 & 262.32 \\
\hline $\mathrm{Nb}$ & 54.17 & 93.1 & 99.3 & 31.1 & 75.97 & 81.06 & 76.25 \\
\hline Mo & 1.61 & 5 & 3.61 & 1.68 & 2.94 & 4.44 & 2.91 \\
\hline In & 0.12 & 0.14 & 0.08 & 0.11 & 0.09 & 0.18 & 0.09 \\
\hline $\mathrm{Sb}$ & 0.09 & 0.11 & 0.08 & 0.09 & 0.09 & 0.12 & 0.07 \\
\hline Cs & 0.42 & 1.25 & 1.1 & 0.48 & 0.76 & 0.98 & 0.77 \\
\hline $\mathrm{Ba}$ & 495.36 & 867.87 & 720.5 & 333.45 & 633.62 & 838.08 & 642.14 \\
\hline Hf & 5.67 & 8.21 & 8.29 & 4.22 & 6.19 & 6.93 & 6.32 \\
\hline $\mathrm{Ta}$ & 3.28 & 6.84 & 6.09 & 1.95 & 4.51 & 5.91 & 4.61 \\
\hline W & 1.03 & 1.5 & 1.24 & 0.5 & 1.04 & 1.47 & 1.1 \\
\hline $\mathrm{Tl}$ & 0.11 & 0.07 & 0.04 & 0.11 & 0.04 & 0.07 & 0.05 \\
\hline $\mathrm{Pb}$ & 4.72 & 6.2 & 4.09 & 2.76 & 3.59 & 5.67 & 3.86 \\
\hline $\mathrm{Bi}$ & 0.02 & 0.02 & 0.04 & 0.02 & 0.06 & 0.04 & 0.02 \\
\hline Th & 7.71 & 9.52 & 8.51 & 3.11 & 6.57 & 8.37 & 6.82 \\
\hline $\mathrm{U}$ & 1.44 & 2.65 & 1.96 & 0.82 & 1.47 & 2.4 & 1.55 \\
\hline
\end{tabular}

Note, (1) $\mathrm{Mg} \#=100 \times \mathrm{Mg} /(\mathrm{Mg}+\mathrm{Fe}), \mathrm{Fe}^{2+} / \mathrm{Fe}^{\text {total }}=0.8998$, cation ratio; (2) $\mathrm{LOI}=$ loss on ignition.

Table 2. Electron probe analyses of olivines in the basaltic rocks from Leiqiong Area and Kontum Area.

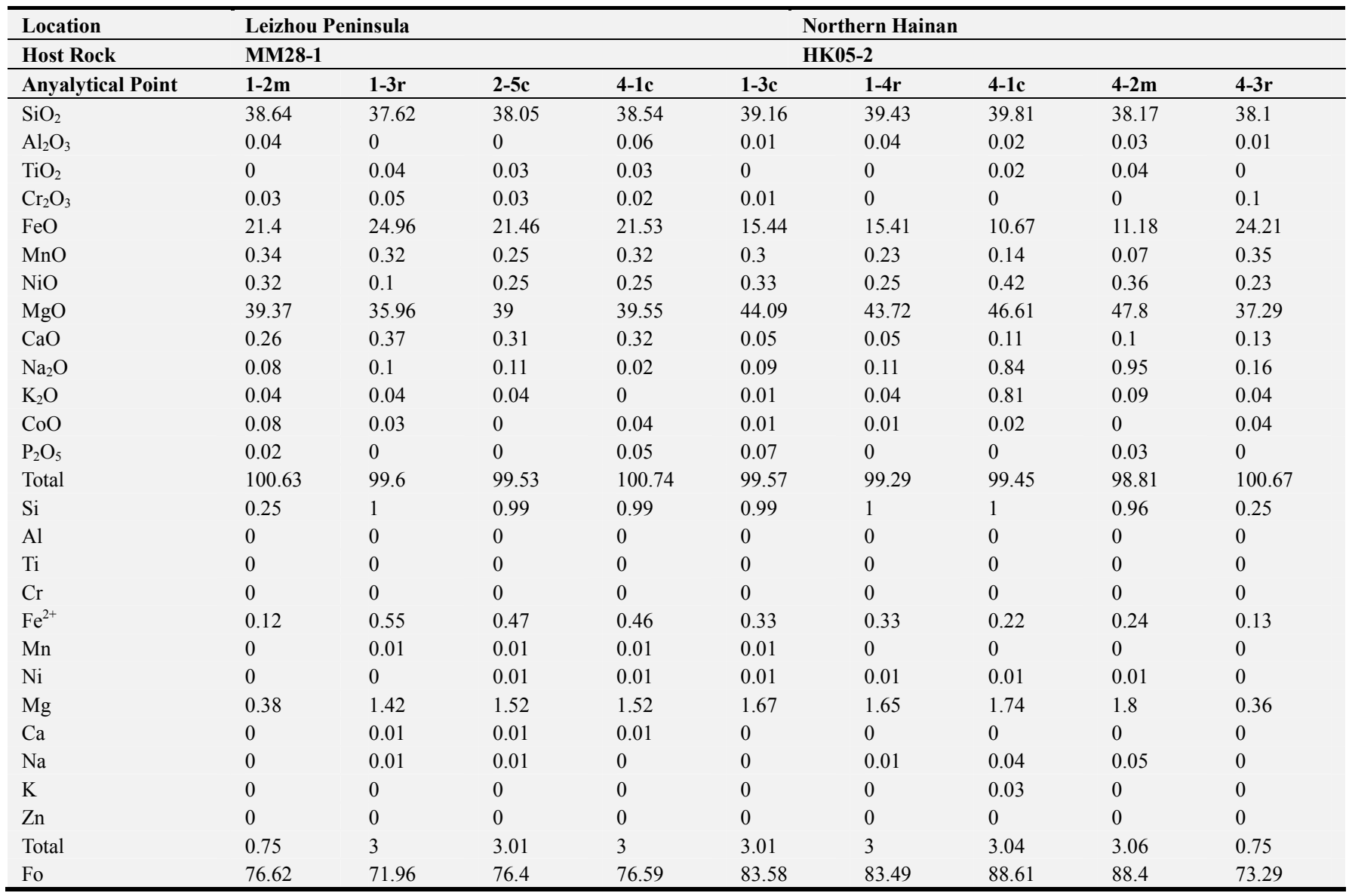


Table 2. Continued.

\begin{tabular}{|c|c|c|c|c|c|c|c|c|c|c|c|c|c|}
\hline \multirow{2}{*}{$\begin{array}{l}\text { Location } \\
\text { Host Rock }\end{array}$} & \multicolumn{4}{|c|}{ Northern Hainan } & \multicolumn{9}{|c|}{ Kontum Area } \\
\hline & HK05-7 & & & & VN23- & & & & VN24-1 & & & & VN25-1 \\
\hline Anyalytical Point & $2-1 c$ & $2-2 m$ & $2-3 r$ & $4-1 c$ & $1-1 c$ & $1-2 \mathrm{r}$ & $2-1 c$ & $5-1 \mathrm{c}$ & $1-1 \mathrm{~m}$ & $1-2 \mathrm{r}$ & $2 b-2 c$ & $2 b-3 r$ & $1-3 c$ \\
\hline $\mathrm{SiO}_{2}$ & 40.71 & 40.25 & 37.77 & 41 & 38.65 & 37.38 & 40.67 & 40.8 & 37.9 & 36.44 & 37.76 & 36.15 & 40.38 \\
\hline $\mathrm{Al}_{2} \mathrm{O}_{3}$ & 0.01 & 0 & 0.02 & 0.01 & 0.02 & 0.04 & 0 & 0.01 & 0.01 & 0 & 0.02 & 0 & 0.02 \\
\hline $\mathrm{TiO}_{2}$ & 0 & 0.08 & 0 & 0.01 & 0.06 & 0.04 & 0 & 0 & 0 & 0.12 & 0.02 & 0 & 0 \\
\hline $\mathrm{Cr}_{2} \mathrm{O}_{3}$ & 0.04 & 0 & 0 & 0 & 0 & 0.04 & 0.01 & 0 & 0.11 & 0.07 & 0.02 & 0.14 & 0.03 \\
\hline $\mathrm{FeO}$ & 9.22 & 9.15 & 23.25 & 8.72 & 23.57 & 24.39 & 10.33 & 11.34 & 24.09 & 30.34 & 24.01 & 31.89 & 10.58 \\
\hline $\mathrm{MnO}$ & 0.21 & 0.09 & 0.34 & 0.14 & 0.28 & 0.45 & 0.2 & 0.25 & 0.31 & 0.45 & 0.36 & 0.43 & 0.12 \\
\hline $\mathrm{NiO}$ & 0.44 & 0.47 & 0.31 & 0.44 & 0.03 & 0.24 & 0.48 & 0.44 & 0.17 & 0.1 & 0.31 & 0.15 & 0.45 \\
\hline $\mathrm{MgO}$ & 49.8 & 48.47 & 37.16 & 48.77 & 36.57 & 35.53 & 48.25 & 47.79 & 37.34 & 31.12 & 37.49 & 29.88 & 48.15 \\
\hline $\mathrm{CaO}$ & 0.07 & 0.08 & 0.2 & 0.07 & 0.28 & 0.41 & 0.07 & 0.04 & 0.27 & 0.33 & 0.26 & 0.34 & 0.1 \\
\hline $\mathrm{Na}_{2} \mathrm{O}$ & 0.06 & 0.08 & 0.28 & 0.09 & 0.24 & 0.62 & 0.34 & 0.04 & 0.04 & 0.05 & 0.15 & 0.1 & 0.05 \\
\hline $\mathrm{K}_{2} \mathrm{O}$ & 0 & 0.04 & 0.08 & 0.04 & 0.02 & 0.14 & 0.09 & 0.02 & 0.01 & 0.02 & 0.04 & 0.02 & 0.01 \\
\hline $\mathrm{CoO}$ & 0.01 & 0 & 0.02 & 0.11 & 0.02 & 0 & 0.03 & 0 & 0.03 & 0.02 & 0 & 0.11 & 0.14 \\
\hline $\mathrm{P}_{2} \mathrm{O}_{5}$ & 0 & 0 & 0.04 & 0 & 0.1 & 0.06 & 0.02 & 0 & 0.09 & 0.02 & 0.12 & 0.07 & 0.02 \\
\hline Total & 100.56 & 98.7 & 99.47 & 99.39 & 99.82 & 99.34 & 100.5 & 100.73 & 100.36 & 99.07 & 100.55 & 99.28 & 100.04 \\
\hline $\mathrm{Si}$ & 1.49 & 1.5 & 1.5 & 1.51 & 1.01 & 1 & 1 & 1 & 0.25 & 1.5 & 1.49 & 1.5 & 1.5 \\
\hline $\mathrm{Al}$ & 0 & 0 & 0 & 0 & 0 & 0 & 0 & 0 & 0 & 0 & 0 & 0 & 0 \\
\hline $\mathrm{Ti}$ & 0 & 0 & 0 & 0 & 0 & 0 & 0 & 0 & 0 & 0 & 0 & 0 & 0 \\
\hline $\mathrm{Cr}$ & 0 & 0 & 0 & 0 & 0 & 0 & 0 & 0 & 0 & 0 & 0 & 0 & 0 \\
\hline $\mathrm{Fe}^{2+}$ & 0.28 & 0.28 & 0.77 & 0.27 & 0.52 & 0.54 & 0.21 & 0.23 & 0.13 & 1.04 & 0.79 & 1.11 & 0.33 \\
\hline $\mathrm{Mn}$ & 0.01 & 0 & 0.01 & 0 & 0.01 & 0.01 & 0 & 0.01 & 0 & 0.02 & 0.01 & 0.02 & 0 \\
\hline $\mathrm{Ni}$ & 0.01 & 0.01 & 0.01 & 0.01 & 0 & 0.01 & 0.01 & 0.01 & 0 & 0 & 0.01 & 0.01 & 0.01 \\
\hline $\mathrm{Mg}$ & 2.71 & 2.69 & 2.19 & 2.68 & 1.43 & 1.41 & 1.77 & 1.75 & 0.36 & 1.91 & 2.2 & 1.85 & 2.66 \\
\hline $\mathrm{Ca}$ & 0 & 0 & 0.01 & 0 & 0.01 & 0.01 & 0 & 0 & 0 & 0.01 & 0.01 & 0.01 & 0 \\
\hline $\mathrm{Na}$ & 0 & 0.01 & 0.02 & 0.01 & 0.01 & 0.03 & 0.02 & 0 & 0 & 0 & 0.01 & 0.01 & 0 \\
\hline K & 0 & 0 & 0 & 0 & 0 & 0 & 0 & 0 & 0 & 0 & 0 & 0 & 0 \\
\hline $\mathrm{Zn}$ & 0 & 0 & 0 & 0 & 0 & 0 & 0 & 0 & 0 & 0 & 0 & 0 & 0 \\
\hline Total & 4.51 & 4.5 & 4.52 & 4.49 & 2.99 & 3.02 & 3.01 & 3 & 0.75 & 4.5 & 4.52 & 4.5 & 4.51 \\
\hline Fo & 90.59 & 90.42 & 74.01 & 90.88 & 73.44 & 72.19 & 89.27 & 88.24 & 73.42 & 64.63 & 73.56 & 62.53 & 89.02 \\
\hline
\end{tabular}

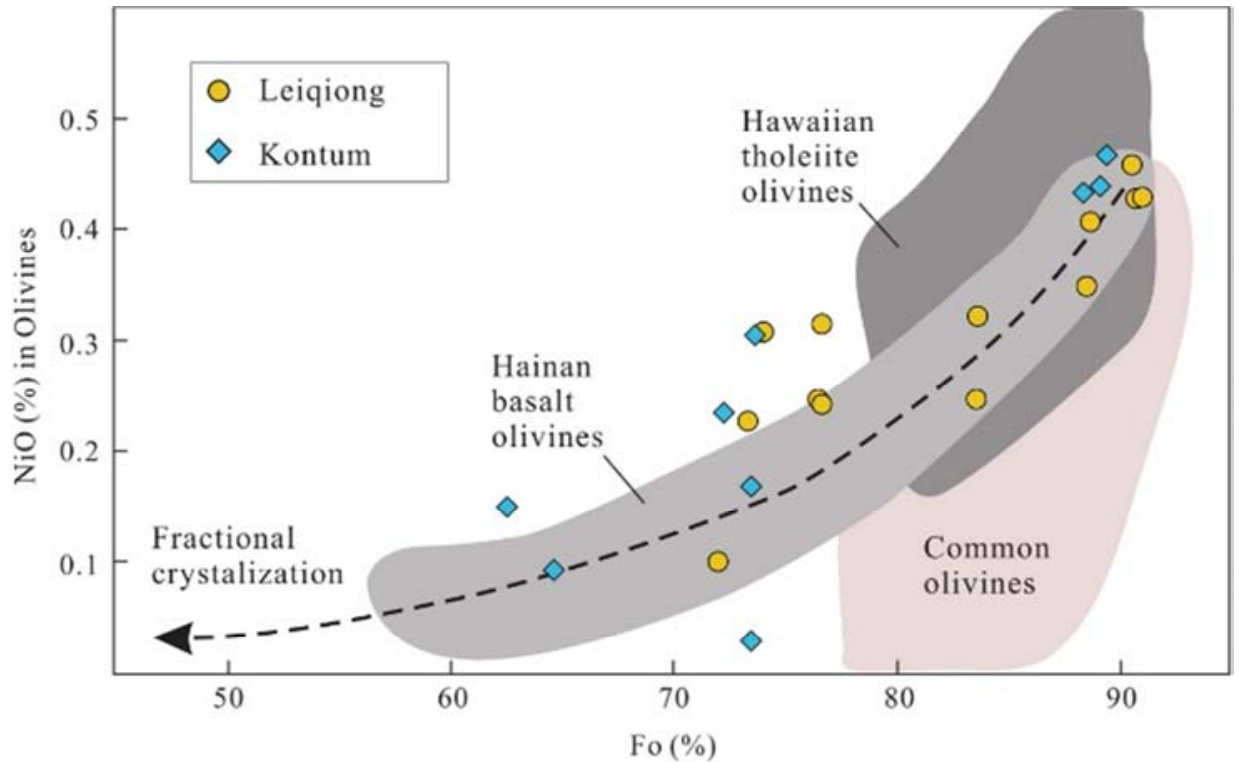

Figure 4. Variations in the composition of olivine phenocrysts. The fractional crystallization trend is from [39]. The field 'common olivines' outlines the compositional range of olivines from peridotite xenoliths, orogenic massifs and ophiolites, oceanic abyssal basalts, and MORB [40], whereas 'Hawaiian tholeiite olivines' denotes the range of olivines from Hawaiian tholeiite basalts[40] and 'Hainan basalt olivines' is based on [15].

In figure 5, the Fo values of olivines are shown as a function of $\mathrm{Mg} \#$ of the host basaltic rocks. The Fe-Mg exchange partition coefficients between the olivine and the basaltic melt

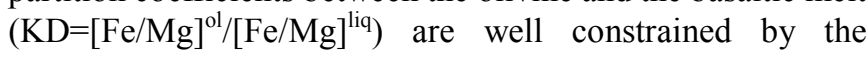
experiments, varying from 0.30 at 1 atm to $0.31-0.34$ at 5-15 kbar [41]. No olivine falls within the equilibrium. Olivine in VN024-1 and MM28-1 are significantly lower than the equilibrium, suggesting the analysed olivine are lately crystallizing matrix grain. Samples HN05-2, VN25-1, VN23-1 and HN05-1 contains olivine above the equilibrium field, indicating that they formed early in the process of magma evolution. The highest Fo values in the Leiqiong and Kontum Areas are 90.88 and 89.27, indicating the $\mathrm{Mg} \#$ of 75 and 71.5 are required for olivine-liquid equilibrium (Figure 5). 


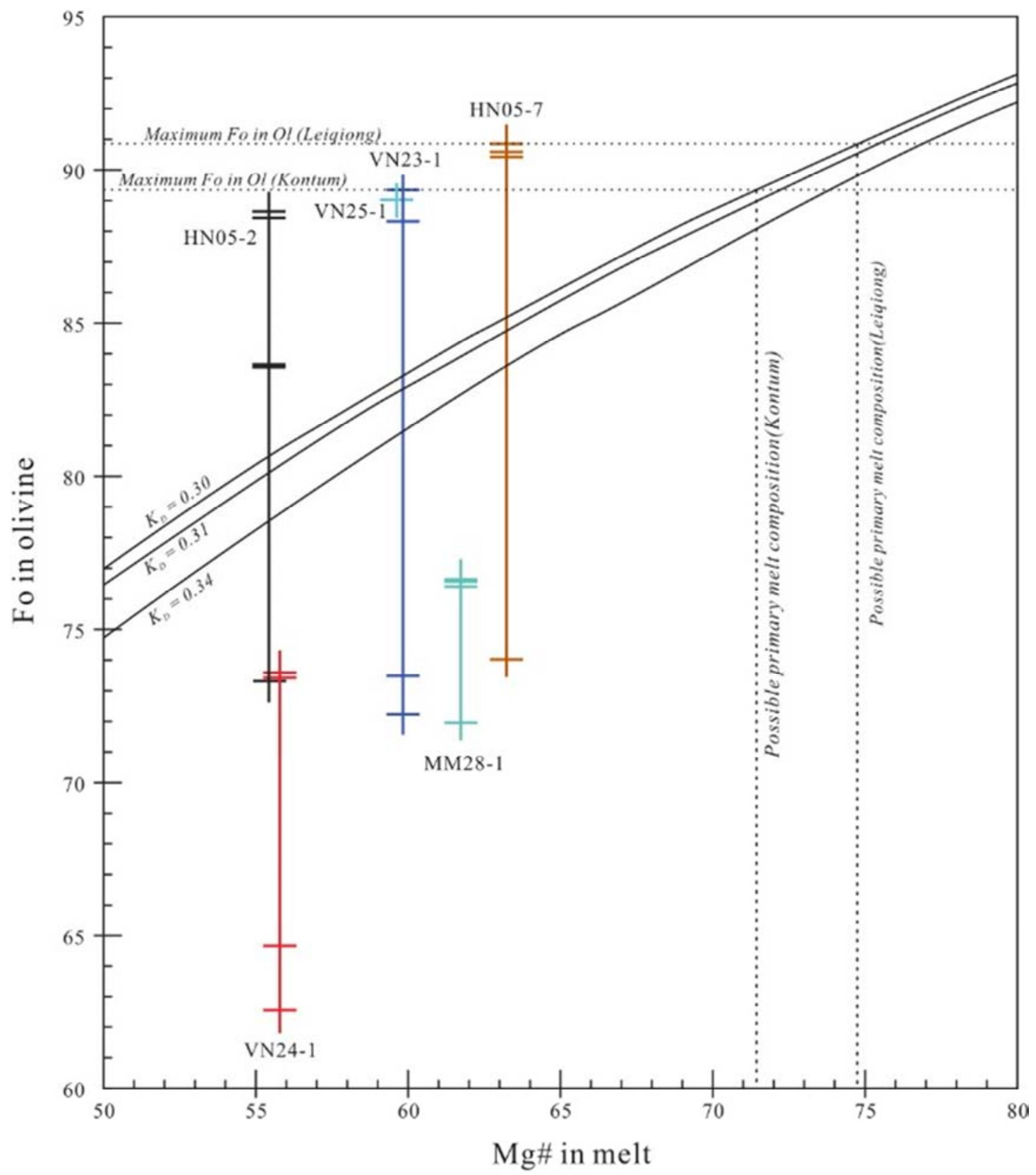

Figure 5. Whole-rock $\mathrm{Mg} \#$ vs. Fo value of olivine. $\mathrm{Mg} \#=100 * \mathrm{Mg} /\left(\mathrm{Mg}+\mathrm{Fe}^{2+}\right)$, calculated assuming $\mathrm{Fe}^{2+} / \mathrm{Fe}^{\text {total }}=0.9$ for whole-rock and total Fe as $\mathrm{Fe} e^{2+}$ in olivine.

\section{Discussion}

\subsection{Possible Crustal Contamination}

The composition may be modified by crustal contamination during the ascent and migration of primary mantle magmas. The effects of crustal contamination can be evaluated by trace elements [42-43]. The Leiqiong basaltic rocks have a relatively variable $\mathrm{Ce} / \mathrm{Pb}$ ratios (6.71-16.33, with an average of 9.52) and $\mathrm{Nb} / \mathrm{U}$ ratios $(26.30-65.58$, with an average of 36.43), which is substantially higher than those of continental crust $(\mathrm{Ce} / \mathrm{Pb}=3.91$ and $\mathrm{Nb} / \mathrm{U}=6.15$; [42-43]) (Figures 6a, 6b). Most of samples are correlated with previous reported samples from the continental margin of northern SCS [44]. Some authors have reported crustal contamination for Vietnamese basalts $[13,16]$. However, the $\mathrm{Ce} / \mathrm{Pb}$ ratios and $\mathrm{Nb} / \mathrm{U}$ ratios of our samples, with yield values range from 13.94-29.80 (average 22.63) and 33.72-50.67 (average 42.26), are obviously higher than those of primitive mantle $(\mathrm{Ce} / \mathrm{Pb}=9$ and $\mathrm{Nb} / \mathrm{U} \approx 30$; [45]) and generally within the range of oceanic basalts (47 \pm 10 ; [45-46]) (Figures 6a, 6b), suggesting limited crustal contamination. In addition, the lack of strong negative $\mathrm{Nb}$ or Ta anomalies in trace element spider diagram (Figure 3a) support the evidence of negligible continental crustal contamination [20-21, 47]. In conclusion, the characteristics of trace element suggest that crustal contamination of our basaltic rocks from two areas are negligible, and these rocks were therefore likely derived from mantle sources. 
a

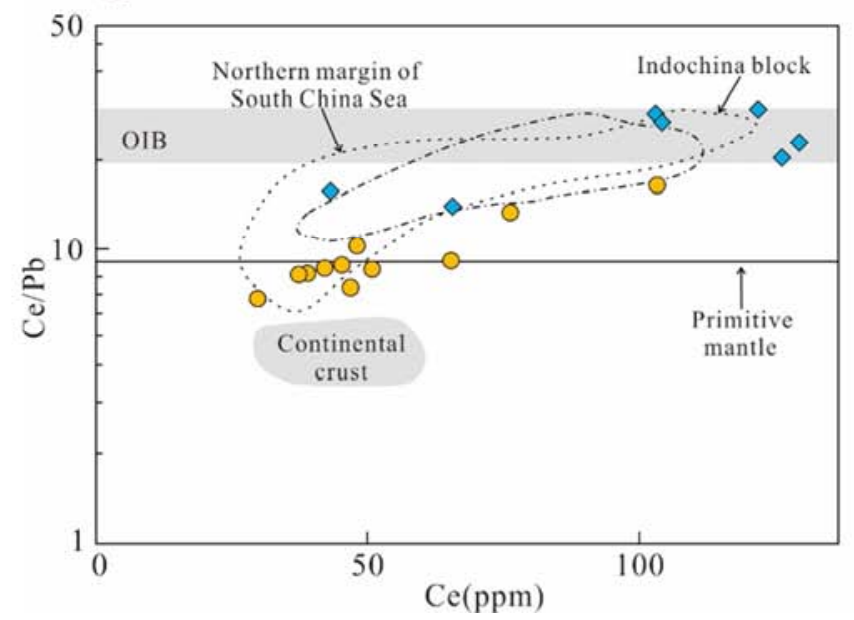

b

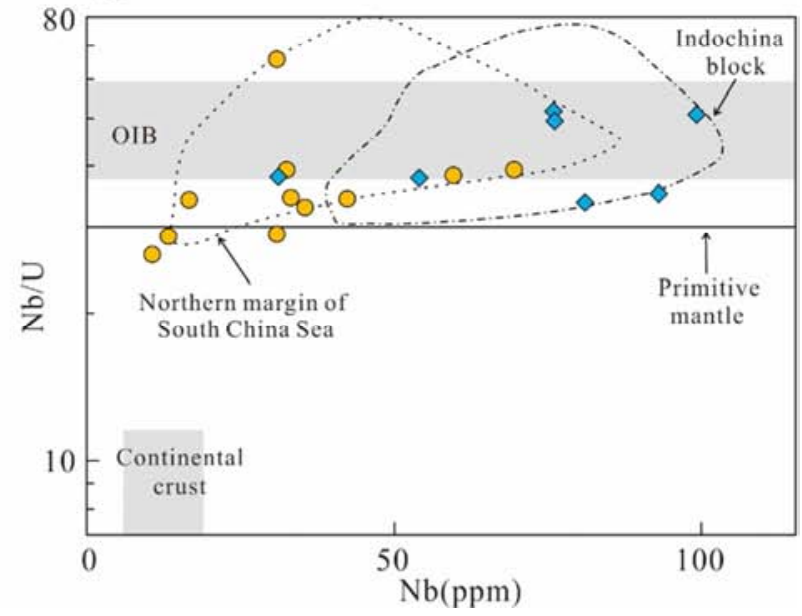

Figure 6. Plots of $\mathrm{Ce} / \mathrm{Pb} v \mathrm{vs}$. $\mathrm{Ce}(\mathrm{a})$ and $\mathrm{Nb} / \mathrm{Uvs}$. $\mathrm{Nb}$ (b) for the basaltic rocks from the Leeiqiong and Kontum Areas. Data for primitive mantle and oceanic island basalt (OIB) are from [45], and data for continental crust (CC) is from [42]. Data for the Northern margin of SCS and the Indochina Block are from [44]. Values in Y-axis is logarithmic scale.
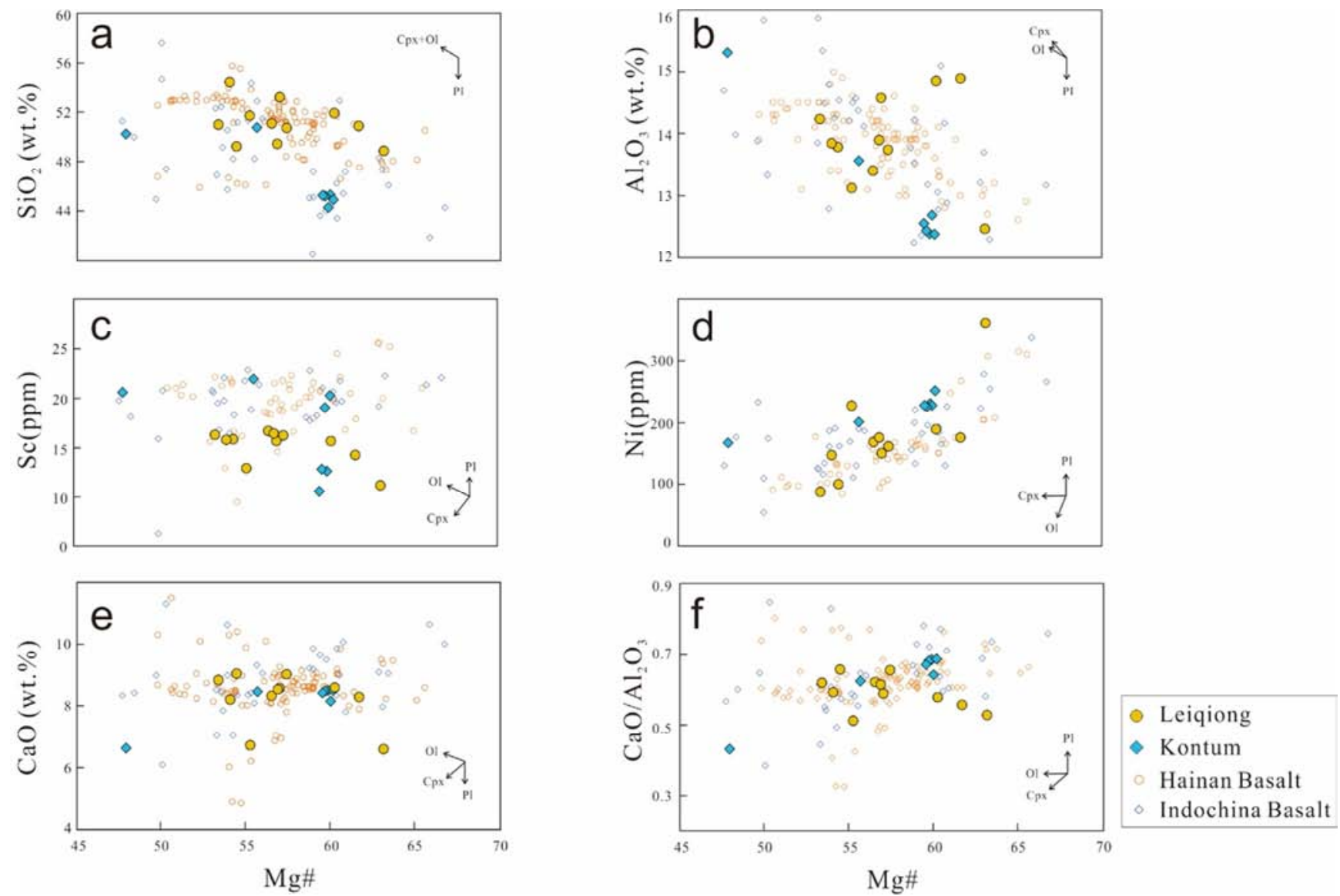

Figure 7. Variation diagram of Mg\# versus selected Major, trace element, and element ratios for basaltic rocks from Leiqiong and Kontum Areas.

\subsection{Fractional Crystallization}

Fractional crystallization is an important process of magma evolution. In order to better observe the fractional crystallization processes, we combined our samples with previous reported samples in the $\mathrm{Mg} \#$ correlation diagrams (Figure 7). For our basaltic rocks, both from the Leiqiong and Kontum Areas, with the decreasing of $\mathrm{Mg} \#$ values, $\mathrm{SiO}_{2}$, $\mathrm{Al}_{2} \mathrm{O}_{3}$ and $\mathrm{Sc}$ are increased (Figures $7 \mathrm{a}, 7 \mathrm{~b}, 7 \mathrm{c}$ ), whereas $\mathrm{Ni}$ is decreased (Figure 7d), no systematic variations occurred in $\mathrm{CaO}, \mathrm{CaO} / \mathrm{Al}_{2} \mathrm{O}_{3}$ (Figures $7 \mathrm{e}, 7 \mathrm{f}$ ). The olivine fractionation increases contents of $\mathrm{SiO}_{2}, \mathrm{Al}_{2} \mathrm{O}_{3}$ and strongly incompatible elements, the olivine+clinopyroxene fractionation decrease the contents of $\mathrm{CaO}$ and $\mathrm{Ni}$, the 
olivine+plagioclase decrease the contents of $\mathrm{Al}_{2} \mathrm{O}_{3}$. The pattern of $\mathrm{SiO}_{2}, \mathrm{Al}_{2} \mathrm{O}_{3}$, and $\mathrm{Ni}$ indicates some fractional crystallization of olivine or olivine+clinopyroxene. $\mathrm{Ca}$ is compatible in clinopyroxene, of which fractionation decreases $\mathrm{CaO}$ contents. The nearly constant of $\mathrm{CaO}$ and $\mathrm{CaO} / \mathrm{Al}_{2} \mathrm{O}_{3}$ suggest clinopyroxene may not be the main fractional phase. The Leiqiong basaltic rocks show slightly $\mathrm{Eu}$ anomalies, indicating that the crystallization of plagioclase. However, the Kontum basaltic rocks shown no $\mathrm{Eu}$ anomalies, indicating the insignificance of plagioclase fractional crystallization. In summary, samples from the Leiqiong and Kontum Areas have mainly undergone the fractional crystallization of olivine, accompanied by the relatively weak fractional crystallization of clinopyroxene and very limited plagioclase fractionation. In addition, samples with highest $\mathrm{Mg \#}$ values can be regarded as relatively primitive magmas which is closed to primary melts.

\subsection{Estimation of Mantle Potential Temperature}

The basaltic rocks produced by mantle plume usually shown some OIB-like features, while the primary melt composition is different from MORB. However, OIB-like characteristics are not always produced by mantle plume. For example, the formation of OIB-like basaltic seamount in East Pacific Rise were independent of mantle plume [48]. In order to constrain the mantle plume, the excess temperature $\left(\mathrm{T}_{e x}\right)$ was proposed to identify the thermal anomalies in the mantle. Meanwhile, $\mathrm{T}_{e x}$ in regimes affected by mantle plume are 160 $300^{\circ} \mathrm{C}$ demonstrated in different calculations [49-51]. In this section, the estimates of the $\mathrm{T}_{p}$ and $\mathrm{T}_{e x}$ in the Leiqiong and Kontum Areas were made in this study.

\subsubsection{Primary Melt Composition}

The basaltic rocks from both the Leiqiong and Kontum Areas had experienced olivine and clinopyroxene fractional crystallization. In order to calculate the primary melt compositions, we selected samples with high $\mathrm{Mg \#} \mathrm{(high}$ $\mathrm{MgO}$ ), as basaltic rocks with lower values are more likely to experienced clinopyroxene and plagioclase fractionation which are unsuitable for the calculation.

For the Leiqiong basaltic rocks, effect of clinopyroxene fractionation on samples with $\mathrm{Mg} \#>61 \quad(\mathrm{MgO} \approx 8.5 \%)$ is negligible [15], allowing us to use MM028-1 (from the Leizhou Peninsula) and HK005-7 (from the Northern Hainan) as the starting composition. For the Kontum basaltic rocks, samples with $\mathrm{MgO}>9 \%$ were chosen for the starting materials [20].

The compositions of primary magmas can be reconstructed by stepwise additions of olivine to computed melts (olivine mass fractions $=0.01$ ), with the compositions of added olivine being calculated based on the $\mathrm{Fe}-\mathrm{Mg}$ exchange coefficient between olivine and silicate melt $(\mathrm{KD}=0.3$; [28]). Repeated addition of olivine was continued until the calculated melt had the composition in equilibrium with olivine as well as reaching the highest Fo values measured for olivine phenocrysts: 90.88 for the Leiqiong basaltic rocks and 89.27 for the Kontum basaltic rocks (Table 2).

The primary melt compositions were calculated by PRIMELT 3 MEGA software [52]. $\mathrm{H}_{2} \mathrm{O}$ content concentrations in the magmas were estimated by the primary Ce contents, assuming that the same $\mathrm{H}_{2} \mathrm{O} / \mathrm{Ce}$ ratios of $\sim 200$ as oceanic basalts [29]. The results of primary melt composition are listed in Table 3.

Table 3. Estimated primary melt composition and mantle potential temperatures.

\begin{tabular}{|c|c|c|c|c|c|c|c|}
\hline Location & Leizhou Peninsula & Northern Hainan & Kontum & & & & \\
\hline Starting sample & MM28-1 & HK05-7 & VN23-1 & VN23-2 & VN25-1 & VN25-2 & VN25-3 \\
\hline$\% \mathrm{Ol}$ addition & 18.00 & 19.00 & 14.00 & 15.00 & 16.00 & 15.00 & 16.00 \\
\hline $\mathrm{SiO}_{2}$ & 49.44 & 48.37 & 45.20 & 45.93 & 45.56 & 45.50 & 45.62 \\
\hline $\mathrm{TiO}_{2}$ & 1.50 & 1.77 & 2.72 & 2.62 & 2.60 & 2.58 & 2.61 \\
\hline $\mathrm{Al}_{2} \mathrm{O}_{3}$ & 12.56 & 10.58 & 11.19 & 11.31 & 10.91 & 11.00 & 11.02 \\
\hline $\mathrm{Cr}_{2} \mathrm{O}_{3}$ & 0.00 & 0.00 & 0.00 & 0.00 & 0.00 & 0.00 & 0.00 \\
\hline $\mathrm{Fe}_{2} \mathrm{O}_{3}$ & 0.87 & 1.02 & 1.17 & 1.13 & 1.17 & 1.18 & 1.16 \\
\hline $\mathrm{FeO}$ & 8.82 & 10.13 & 11.08 & 10.82 & 11.23 & 11.20 & 11.19 \\
\hline $\mathrm{MnO}$ & 0.15 & 0.17 & 0.17 & 0.18 & 0.18 & 0.18 & 0.18 \\
\hline $\mathrm{MgO}$ & 14.91 & 17.13 & 14.94 & 15.07 & 15.62 & 15.49 & 15.52 \\
\hline $\mathrm{CaO}$ & 7.00 & 5.60 & 7.70 & 7.29 & 7.46 & 7.60 & 7.42 \\
\hline $\mathrm{Na}_{2} \mathrm{O}$ & 2.94 & 2.57 & 2.47 & 2.16 & 2.31 & 2.35 & 2.30 \\
\hline $\mathrm{K}_{2} \mathrm{O}$ & 1.46 & 1.96 & 2.51 & 2.64 & 2.14 & 2.09 & 2.15 \\
\hline $\mathrm{NiO}$ & 0.00 & 0.00 & 0.00 & 0.00 & 0.00 & 0.00 & 0.00 \\
\hline $\mathrm{P}_{2} \mathrm{O}_{5}$ & 0.37 & 0.70 & 0.85 & 0.83 & 0.81 & 0.84 & 0.82 \\
\hline $\mathrm{H}_{2} \mathrm{O}$ & 0.77 & 1.69 & 2.06 & 1.99 & 1.68 & 2.11 & 1.70 \\
\hline$F(\%)$ & 14.47 & 16.75 & 0.08 & 2.83 & 3.00 & 2.53 & 2.90 \\
\hline $\mathrm{P}_{\mathrm{ol}}{ }^{1}$ & 14.94 & 19.73 & 27.01 & 24.60 & 26.80 & 26.82 & 26.44 \\
\hline $\mathrm{P}_{\mathrm{ol}}{ }^{2}$ & 15.57 & 20.24 & 34.14 & 30.95 & 32.56 & 32.84 & 32.33 \\
\hline $\mathrm{P}_{\mathrm{ol}}{ }^{3}$ & 18.96 & 23.52 & 24.05 & 22.92 & 24.05 & 23.69 & 23.78 \\
\hline $\mathrm{P}_{\mathrm{ol}}^{\text {avg }}$ & 16.49 & 21.16 & 28.40 & 26.15 & 27.80 & 27.78 & 27.52 \\
\hline $\mathrm{T}_{\mathrm{ol}}{ }^{1}$ & 1432.35 & 1492.00 & 1465.73 & 1463.19 & 1479.62 & 1476.96 & 1476.84 \\
\hline $\mathrm{T}_{\mathrm{ol}}{ }^{2}$ & 1427.71 & 1491.61 & 1440.69 & 1440.82 & 1456.49 & 1448.58 & 1453.25 \\
\hline $\mathrm{T}_{\mathrm{ol}}{ }^{\text {avg }}$ & 1430.03 & 1491.81 & 1453.21 & 1452.01 & 1468.05 & 1462.77 & 1465.04 \\
\hline $\mathrm{T}_{\mathrm{p}}{ }^{1}$ & 1453.43 & 1507.60 & 1454.18 & 1457.74 & 1471.61 & 1468.34 & 1469.22 \\
\hline $\mathrm{T}_{\mathrm{p}}^{2}$ & 1445.00 & 1554.03 & 1644.86 & 1619.73 & 1658.33 & 1656.24 & 1654.18 \\
\hline
\end{tabular}




\begin{tabular}{llllllll}
\hline Location & Leizhou Peninsula & Northern Hainan & Kontum Area & & & \\
\hline Starting sample & MM28-1 & HK05-7 & VN23-1 & VN23-2 & VN25-1 & VN25-2 & VN25-3 \\
\hline $\mathrm{T}_{\mathrm{p}}{ }^{3}$ & 1524.21 & 1603.29 & 1441.24 & 1459.71 & 1476.52 & 1465.43 & 1472.61 \\
$\mathrm{~T}_{\mathrm{p}}{ }^{\text {avg }}$ & 1474.21 & 1554.97 & 1513.43 & 1512.39 & 1535.49 & 1530.00 & 1532.00 \\
\hline
\end{tabular}

$\mathrm{F}$ is degree of melting, was estimated by Eq. (A2) of [53].

$\mathrm{P}_{\mathrm{ol}}{ }^{1}$ to $\mathrm{P}_{\mathrm{ol}}{ }^{3}$ are effective melting pressures in kbar. $\mathrm{P}_{\mathrm{ol}}{ }^{1}$ is as defined by Eq. (42) of [28]; $\mathrm{P}_{\mathrm{ol}}{ }^{2}$ as defined by [54]; $\mathrm{P}_{\mathrm{ol}}{ }^{3}$ as defined by [55]; $\mathrm{P}_{\mathrm{ol}}{ }^{\text {avg }}$ is the average of $\mathrm{P}_{\mathrm{ol}}{ }^{1}$ to $\mathrm{P}_{\mathrm{ol}}{ }^{3}$.

$\mathrm{T}_{\mathrm{ol}}{ }^{1}$ and $\mathrm{T}_{\mathrm{ol}}{ }^{2}$ are the melting temperatures $\left({ }^{\circ} \mathrm{C}\right)$ of the melt segregation: $\mathrm{T}_{\mathrm{ol}}{ }^{1}$ is according to Eq. (14) of [28]; $\mathrm{T}_{\mathrm{ol}}{ }^{2}$ according to [53]; $\mathrm{T}_{\mathrm{ol}}{ }^{\text {avg }}$ is the average of $\mathrm{T}_{\mathrm{ol}}{ }^{1}$ to $\mathrm{T}_{\mathrm{ol}}{ }^{2}$.

$\mathrm{T}_{\mathrm{p}}{ }^{1}$ and $\mathrm{T}_{\mathrm{p}}{ }^{2}$ are the mantle potential temperatures $\left({ }^{\circ} \mathrm{C}\right): \mathrm{T}_{\mathrm{p}}{ }^{1}$ is estimated using the $\mathrm{MgO}$ contents in the primary melts following [29]; $\mathrm{T}_{\mathrm{p}}{ }^{2}$ is estimated following olivine-liquid equilibria thermometry in [51]; $\mathrm{T}_{\mathrm{p}}{ }^{\text {avg }}$ is the average of $\mathrm{T}_{\mathrm{p}}{ }^{1}$ to $\mathrm{T}_{\mathrm{p}}{ }^{2}$.

The added olivine for the basaltic rocks from the Leizhou Peninsula, the Northern Hainan, and the Kontum Area are $18 \%, 19 \%$ and $14-16 \%$, respectively. The degree of partial melting $(F)$ of basaltic rocks in Leizhou Peninsula (14.47\%) and Northern Hainan (16.75\%) are similar, within the ranges of previous reported Hainan basalt (11-19\%; [15]). In the Kontum basaltic rocks from Central Vietnam, $F$ ranges from $0.08-3 \%$ (Table 3 ), slightly lower than the basaltic rocks from the Southern Vietnam (4-7\%; [20]).

\subsubsection{Mantle Potential Temperature}

The effective crystallization pressures in the Leiqiong Area (16.49-21.16 kbar) are slightly lower than that in the Kontum Area (26.15-28.40 kbar), where the melting temperatures are in a small range of $1430-1491^{\circ} \mathrm{C}$ (Table 3). Following empirical equations of [36], we used the $\mathrm{MgO}$ contents in primary melt composition to estimate the mantle potential temperature $\left(\mathrm{T}_{p}\right)$. The results in the Leizhou Peninsula, the Northern Hainan and the Kontum Area are $1453^{\circ} \mathrm{C}, 1507^{\circ} \mathrm{C}$ and $1454-1472^{\circ} \mathrm{C}$, respectively. In order to be more confident for the calculated results, we further estimated $\mathrm{T}_{p}$ from olivine-liquid equilibration thermometer for comparison [51]. The estimated $\mathrm{T}_{p}$ we obtained in the Leizhou Peninsula, the Northern Hainan, and the Kontum Area are $1524^{\circ} \mathrm{C}, 1603^{\circ} \mathrm{C}$, and $1441-1476^{\circ} \mathrm{C}$, respectively. Comparing the two methods, the average $\mathrm{T}_{p}$ is defined as the results, of which value beneath the Leizhou Peninsula, the Northern Hainan and the Kontum Area are $1489^{\circ} \mathrm{C}, 1555^{\circ} \mathrm{C}$ and $1458 \pm 2.6^{\circ} \mathrm{C}$ (weighted average).

The $\mathrm{T}_{p}$ estimated from SCS MORB is around $1380^{\circ} \mathrm{C}$ [9], which is comparable with global MORs $\left(1280-1450^{\circ} \mathrm{C},[28]\right)$ Using $\mathrm{T}_{p}$ of SCS MORB as the background of upper mantle temperature under the SCS region, we obtained the $\mathrm{T}_{e x}$ beneath the studied areas, which shows in a range of $\sim 78$ $175^{\circ} \mathrm{C}$. Compared in typical plume affected areas, the $\mathrm{T}_{e x}$ of Hawaii (Mauna Kea) and Iceland are $220^{\circ} \mathrm{C}$ and $\sim 170^{\circ} \mathrm{C}$, respectively [51]; Moreover, the $\mathrm{T}_{e x}$ estimated from basaltic rocks in regions of Tristan Plume and Circe Plume are $\sim 160^{\circ} \mathrm{C}$ and $\sim 280^{\circ} \mathrm{C}$ [50]. Although $\mathrm{T}_{e x}$ values varies in different plume, the generally range of $\sim 160-\sim 300^{\circ} \mathrm{C}$ were considered affected by mantle plume. Therefore, the highest $\mathrm{T}_{e x}\left(\sim 175^{\circ} \mathrm{C}\right)$ indicate that the studied area was affected by mantle plume.

\subsubsection{Thermal Structure of the Hainan Plume}

The result of calculated $\mathrm{T}_{p}$ in the Northern Hainan $\left(1555^{\circ} \mathrm{C}\right)$ is consistent with previous reported $\mathrm{T}_{p}$ from Northern Hainan $\left(1521 \pm 24^{\circ} \mathrm{C},[15]\right)$, which increases our confidence of the calculation. $\mathrm{T}_{p}$ is beneath the Northern Hainan is $\sim 65^{\circ} \mathrm{C}$ higher than that of the Leizhou Peninsula $\left(1489^{\circ} \mathrm{C}\right)$, possibly due to the deeper source of the Northern Hainan basaltic rocks. The estimated $\mathrm{T}_{p}$ from the Kontum basaltic rocks range from $1448-1474^{\circ} \mathrm{C}$, while the reported $\mathrm{T}_{p}$ from the Southern Vietnam was in a range of $1470-1480^{\circ} \mathrm{C}$ [20]. The similar results indicate the similar source depth of basaltic rocks from the Central and Southern Vietnam. However, these temperatures are $\sim 90^{\circ} \mathrm{C}$ lower than that in the Northern Hainan. The plume theory suggested that the temperature variability attributed to the thermal structure of the mantle plume. The temperature of a plume head is predicted to be appreciably higher at the center of the head than at its margins [56-57]. Therefore, the higher $\mathrm{T}_{p}$ beneath Northern Hainan may be caused by its position that closer to the center of plume head, whereas $\mathrm{T}_{p}$ under the Leizhou Peninsula, the Central Vietnam and the Southern Vietnam might derive from peripheral regions far from the plume center.

The geophysical investigations reveal the head of Hainan Plume is beneath the northern part of Hainan Island [23-24], which is consistent with higher $\mathrm{T}_{p}$ under the Northern Hainan. The tomographic images in South China reveal that the Hainan Plume raises sub-vertically from lower mantle and accumulated in in the mantle transition zone, then diffuses laterally, and divides into multiple branches [26]. The low velocity also extends to the Southern Vietnam [25]. The collision of India with Eurasia might induce the lateral diffuses of lithosphere and reactive Cenozoic fault systems in the Indochina Peninsula [58], revealing the fact that these fault systems maybe promote the magma upwelling branching of from Hainan Plume.

\subsection{Tectonic Signification}

The upwelling of Hainan Plume suggested the acceleration of the development of western Pacific marginal sea basins [59], among which the SCS experienced a nearly complete Wilson cycle [60]. The continental rift stage of the SCS was featured by volcanic basins in South China. The Sanshui Basin is one of a representation, where the volcanism can be divided into earlier phase dominated by trachyte and, the later phase dominated by intraplate basaltic rocks [61]. The intraplate basaltic rocks shown OIB-like characteristics, indicating a mixture of DMM and EM2 mantle source [21], which is similar to the post-spreading basaltic rocks we analyzed. The magmatism in the Sanshui Basin is widely considered to be the consequence of regional asthenosphere upwelling [62-63]. 
The comparison of lithological combination between the Sanshui Basin and the Red Sea Area suggest that the development of the Sanshui Basin is very similar to the Red Sea model, as magmatism in the Sanshui Basin representing the infancy of the SCS [61].

However, in the syn-spreading stage of SCS basin, only limited magmatism reported in the surrounding region of the SCS. The extraction of Indochina Block was induced by the India-Asian collision [64]. This lateral movement of tectonic layers might suspend the magmatic supply and ended the break of continental rift. In this case, only basic magmas sourced from the decompression melting of lithospheric upper mantle upwelling, which formed the oceanic crust of the SCS [65].

The northwards movement of the Philippine Plate was considered to be one of the reasons for the cease of SCS basin spreading [66]. The Philippine Plate counteracted the effect of extraction of Indochina block, which resuscitated the upwelling of deep mantle. The Hainan Plume formed a subvertical column, with plume center beneath Northern Hainan and small conduits branched along fault systems to Indochina Peninsula and South China, producing large scale Late Cenozoic magmatism in the SCS and its surrounding regions.

The magmatism in the pre- and post-spreading stages of the SCS reflect similar mantle source. However, the scale of pre-spreading stage is smaller than post-spreading stage. We hypothesize that the small scaly magmatism in pre-spreading stage of the SCS has reflect on the infancy of mantle upwelling, which is constrained by plate tectonic activities in syn-spreading stage. In the post-spreading stage, with the revoking of tectonic constrains, the ascent of plume-affected deep mantle materials formed large scale magmatism in the SCS and its surroundings, which probably suggest the arising of a new continental rift environment.

\section{Conclusions}

This paper studies the geochemical characteristics of the Late Cenozoic basaltic rocks from Leiqiong Area and Kontum Area in the continental margins of the northern and western SCS, and reaches to the following conclusions:

1. The geochemical characteristics of basaltic rocks from the Leiqiong and Kontum Areas were consistent, featured with OIB-like trace element distribution and REE pattern.

2. The $\mathrm{T}_{p}$ values beneath the Leizhou Peninsula, the Northern Hainan and the Kontum Area are $1489^{\circ} \mathrm{C}$, $1555^{\circ} \mathrm{C}$ and $1458 \pm 2.6^{\circ} \mathrm{C}$, respectively. The $\mathrm{T}_{e x}$ in studied areas range from $\sim 78-175^{\circ} \mathrm{C}$, suggest the influence by mantle plume.

\section{Acknowledgements}

The authors thank Chao Yang for helping us to collect samples. Jingwu Yin is thanked for assistance during EPMA analyses. We thank Dongsheng Wang for his linguistic assistance. We are grateful for the thoughtful and helpful comments by the anonymous reviewers.

\section{References}

[1] Taylor, B.; Hayes, D. E. Origin and history of the South China Sea basin. GMS 1983, 27, 23-56.

[2] Briais, A.; Patriat, P.; Tapponnier, P. Updated interpretation of magnetic anomalies and seafloor spreading stages in the south China Sea: Implications for the Tertiary tectonics of Southeast Asia. Journal of Geophysical Research: Solid Earth 1993, 98, 6299-6328. doi: 10.1029/92jb02280.

[3] Yan, P.; Deng, H.; Liu, H.; Zhang, Z.; Jiang, Y. The temporal and spatial distribution of volcanism in the South China Sea region. Journal of Asian Earth Sciences 2006, 27, 647-659. doi 10.1016/j.jseaes.2005.06.005.

[4] Xu, Y. G.; Wei, J. X.; Qiu, H. N.; Zhang, H. H.; Huang, X. L. Opening and evolution of the South China Sea constrained by studies on volcanic rocks: Preliminary results and a research design. Chinese Science Bulletin 2012, 57, 3150-3164. doi: 10.1007/s11434-011-4921-1.

[5] Chung, S. L.; Cheng, H.; Jahn, B. M.; O'Reilly, S. Y.; Zhu, B. Major and trace element, and $\mathrm{Sr}-\mathrm{Nd}$ isotope constraints on the origin of Paleogene volcanism in South China prior to the South China Sea opening. Lithos 1997, 40, 203-220. doi: 10.1016/s0024-4937(97)00028-5.

[6] Zhu, B. Q.; Wang, H. F.; Chen, Y. W.; Chang, X. Y.; Hu, Y. G.; Xie, J. Geochronological and geochemical constraint on the Cenozoic extension of Cathaysian lithosphere and tectonic evolution of the border sea basins in East Asia. Journal of Asian Earth Sciences 2004, 24, 163-175. doi: 10.1016/j.jseaes.2003.10.006.

[7] Zhang, W.; Fang, N.; Yuan, X.; Cui, L. Geochemical and Mineralogical Investigation on Different Types of Cenozoic Basalts in the Sanshui Basin: Implications for Magma Mixing Processes. Journal of Earth Science 2019, 30, 754-762. doi: 10.1007/s12583-019-1208-y.

[8] Sun, K.; Wu, T.; Liu, X.; Chen, X. G.; Li, C. F. Lithogeochemistry of the Mid-Ocean Ridge Basalts near the Fossil Ridge of the Southwest Sub-Basin, South China Sea. Minerals 2020, 10, 465. doi: 10.3390/min10050465.

[9] Yu, X.; Liu, Z. Non-mantle-plume process caused the initial spreading of the South China Sea. Scientific Reports 2020, 10, 1-10. doi: 10.1038/s41598-020-65174-y.

[10] Flower, M. F.; Zhang, M.; Chen, C. Y.; Tu, K.; Xie, G. Magmatism in the South China Basin. 2. Post-spreading Quaternary basalts from Hainan Island, south China. Chemical Geology 1992, 97, 65-87. doi: 10.1016/0009-2541(92)90136-S.

[11] Zhu, B.; Wang, H. Nd-Sr-Pb isotopic and chemical evidence for the volcanism with MORB-OIB source characteristics in the Leiqiong Area, China. Geochemic 1989, 3, 93-201. doi: 10.19700/j.0379-1726.1989.03.001.

[12] Tu, K.; Flower, M. F.; Carlson, R. W.; Ming Zhang.; Guanghong Xie. $\mathrm{Sr}, \mathrm{Nd}$, and $\mathrm{Pb}$ isotopic compositions of Hainan basalts (south China): implications for a subcontinental lithosphere Dupal source. Geology 1991, 19, 567-569. doi: 10.1130/0091-7613(1991) 019<0567:SNAPIC>2.3.CO;2. 
[13] Hoang, N.; Flower, M. F.; Carlson, R. W. Major, trace element, and isotopic compositions of Vietnamese basalts: Interaction of hydrous EM1-rich asthenosphere with thinned Eurasian lithosphere. Geochimica et Cosmochimica Acta 1996, 60, 4329-4351. doi: 10.1016/S0016-7037(96)00247-5.

[14] Zou, H.; Fan, Q. U-Th isotopes in Hainan basalts: Implications for sub-asthenospheric origin of EM2 mantle endmember and the dynamics of melting beneath Hainan Island. Lithos 2010, 116, 145-152. doi: 10.1016/j.lithos.2010.01.010.

[15] Wang, X. C.; Li, Z. X.; Li, X. H.; Li, J.; Liu, Y.; Long, W. G.; Zhou, J. B.; Wang, F. Temperature, pressure, and composition of the mantle source region of late cenozoic basalts in Hainan Island, SE Asia: A consequence of a young thermal mantle plume close to subduction zones? Journal of Petrology 2012, 53, 177-233. doi: 10.1093/petrology/egr061.

[16] Koszowska, E.; Wolska, A.; Zuchiewicz, W.; Cuong, N. Q.; Pécskay, Z. Crustal contamination of Late Neogene basalts in the Dien Bien Phu Basin, NW Vietnam: Some insights from petrological and geochronological studies. Journal of Asian Earth Sciences 2007, 29, 1-17. doi: 10.1016/j.jseaes.2005.12.003.

[17] Hoàng, N.; Flower, M. F.; o.ng Chí, C. T.; Xuân, P. T.; Quý, H. V.; Son, T. T. Collision-induced basalt eruptions at Pleiku and BuÔn Mê Thuôt, south-central Viet Nam. Journal of Geodynamics 2013, 69, 65-83. doi: 10.1016/j.jog.2012.03.012.

[18] Hoang, N.; Flower, M. Petrogenesis of cenozoic basalts from Vietnam: Implication for origins of a 'diffuse igneous province'. Journal of Petrology 1998, 39, 369-395. doi: 10.1093/petroj/39.3.369.

[19] Wang, X. c.; Li, Z. X.; Li, X. H.; Li, J.; Xu, Y. G.; Li, X. H. Identification of an ancient mantle reservoir and young recycled materials in the source region of a young mantle plume: Implications for potential linkages between plume and plate tectonics. Earth and Planetary Science Letters 2013, 377-378, 248-259. doi: 10.1016/j.eps1.2013.07.003.

[20] An, A. R.; Choi, S. H.; Yu, Y.; Lee, D. C. Petrogenesis of Late Cenozoic basaltic rocks from southern Vietnam. Lithos 2017, 272-273, 192-204. doi: 10.1016/j.lithos.2016.12.008.

[21] Yang, S.; Fang, N. Geochemical variation of volcanic rocks from the South China Sea and neighboring land: Implication for magmatic process and mantle structure. Acta Oceanologica Sinica 2015, 34, 112-124. doi: 10.1007/s13131-015-0759-8.

[22] Zhang, Y.; Yu, K.; Fan, T.; Yue, Y.; Wang, R.; Jiang, W.; Xu, S.; Wang, Y. Geochemistry and petrogenesis of Quaternary basalts from Weizhou Island, northwestern South China Sea: Evidence for the Hainan plume. Lithos 2020, 362-363, 105493. doi: $10.1016 /$ j.lithos.2020.105493.

[23] Lebedev, S.; Nolet, G. Upper mantle beneath Southeast Asia from $\mathrm{S}$ velocity tomography. Journal of Geophysical Research: Solid Earth 2003, 108. doi: 10.1029/2000jb000073.

[24] Montelli, R.; Nolet, G.; Dahlen, F. A.; Masters, G. A catalogue of deep mantle plumes: New results from finitefrequency tomography. Geochemistry, Geophysics, Geosystems 2006, 7. doi: 10.1029/2006GC001248.

[25] Huang, J.; Zhao, D. High-resolution mantle tomography of China and surrounding regions. Journal of Geophysical Research: Solid Earth 2006, 111, 1-21. doi: 10.1029/2005JB004066.
[26] Xia, S.; Zhao, D.; Sun, J.; Huang, H. Teleseismic imaging of the mantle beneath southernmost China: New insights into the Hainan plume. Gondwana Research 2016, 36, 46-56. doi: 10.1016/j.gr.2016.05.003.

[27] McKenzie, D.; Jackson, J.; Priestley, K. Thermal structure of oceanic and continental lithosphere. Earth and Planetary Science Letters 2005, 233, 337-349. doi: 10.1016/j.eps1.2005.02.005.

[28] Putirka, K. D. Thermometers and barometers for volcanic systems. Reviews in Mineralogy and Geochemistry, 2008, 69, 61-120. doi: 10.2138/rmg.2008.69.3.

[29] Herzberg, C.; Asimow, P. D.; Arndt, N.; Niu, Y.; Lesher, C. M.; Fitton, J. G.; Cheadle, M. J.; Saunders, A. D. Temperatures in ambient mantle and plumes: Constraints from basalts, picrites, and komatiites. Geochemistry, Geophysics, Geosystems 2007, 8. doi: 10.1029/2006GC001390.

[30] Campbell, I. H. Identification of ancient mantle plumes. In Mantle plumes: their identification through time; Geological Society of America, 2001. doi: 10.1130/0-8137-2352-3.5.

[31] Xu, Y. G.; He, B.; Huang, X.; Luo, Z.; Chung, S. L.; Xiao, L.; Zhu, D.; Shao, H.; Fan, W. M.; Xu, J.; Wang, Y. J. Identification of mantle plumes in the Emeishan Large Igneous Province. Episodes 2007, 30, 32-42. doi: 10.18814/epiiugs/2007/v30i1/005.

[32] Hoang, T. H. A.; Choi, S. H.; Yu, Y.; Pham, T. H.; Nguyen, K. H.; Ryu, J. S. Geochemical constraints on the spatial distribution of recycled oceanic crust in the mantle source of late Cenozoic basalts, Vietnam. Lithos 2018, 296-299, 382395. doi: 10.1016/j.lithos.2017.11.020.

[33] Irvine, T. N.; Baragar, W. R A. A Guide to the Chemical Classification of the Common Volcanic Rocks. Canadian Journal of Earth Sciences 1971, 8, 523-548. doi: 10.1139/e71-055.

[34] Sun, S. S.; McDonough, W. F. Chemical and isotopic systematics of oceanic basalts: Implications for mantle composition and processes. Geological Society Special Publication 1989, 42, 313 345. doi: 10.1144/GSL.SP.1989.042.01.19.

[35] Frey, F. A.; Green, D. H.; Roy, S. D. Integrated models of basalt petrogenesis: A study of quartz tholeiites to olivine melilitites from South Eastern Australia utilizing geochemical and experimental petrological data. Journal of Petrology 1978, 19, 463-513. doi: 10.1093/petrology/19.3.463.

[36] Wilkinson, J. F.; Maitre, R. W. Upper mantle amphiboles and micas and $\mathrm{TiO}_{2}, \mathrm{~K}_{2} \mathrm{O}$, and $\mathrm{P}_{2} \mathrm{O}_{5}$ abundances and 100 $\mathrm{Mg} / \mathrm{Mg}+\mathrm{Fe}$ ratios of common basalts and andesites: Implications for modal mantle metasomatism and undepleted mantle compositions. Journal of Petrology 1987, 28, 37-73. doi: 10.1093/petrology/28.1.37.

[37] Foley, S. F.; Prelevic, D.; Rehfeldt, T.; Jacob, D. E. Minor and trace elements in olivines as probes into early igneous and mantle melting processes. Earth and Planetary Science Letters 2013, 363, 181-191. doi: 10.1016/j.epsl.2012.11.025.

[38] Thompson, R. N.; Gibson, S. a.; Bay, B. Transient high temperatures in mantle plume heads inferred from magnesian olivines in Phanerozoic picrites. Nature 2000, 407.

[39] Sato, H. Nickel content of basaltic magmas: identification of primary magmas and a measure of the degree of olivine fractionation. Lithos 1977, 10, 113-120. doi: 10.1016/0024-4937(77) 90037-8. 
[40] Sobolev, A. V.; Hofmann, A. W.; Sobolev, S. V.; Nikogosian, I. K. An olivine-free mantle source of Hawaiian shield basalts. Nature 2005, 434, 590-597. doi: 10.1038/nature03411.

[41] Roeder, P. L.; Emslie, R. F. Olivine-liquid equilibrium. Contributions to Mineralogy and Petrology 1970, 29, 275-289. doi: 10.1007/BF00371276.

[42] Rudnick, R.; Gao, S. Composition of the continental crust. The crust 2003, 3, 1-64.

[43] Salters, V. J.; Stracke, A. Composition of the depleted mantle. Geochemistry, Geophysics, Geosystems 2004, 5. doi: 10.1029/2003GC000597.

[44] Yan, Q.; Shi, X.; Metcalfe, I.; Liu, S.; Xu, T.; Kornkanitnan, N.; Sirichaiseth, T.; Yuan, L.; Zhang, Y.; Zhang, H. Hainan mantle plume produced late Cenozoic basaltic rocks in Thailand, Southeast Asia. Scientific Reports 2018, 8, 1-14. doi: 10.1038/s41598-018-20712-7.

[45] Hofmann, A. W. Chemical differentiation of the Earth: the relationship between mantle, continental crust, and oceanic crust. Earth and Planetary Science Letters 1988, 90, 297-314. doi: 10.1016/0012-821X(88)90132-X.

[46] Niu, Y.; O'Hara, M. J. Origin of ocean island basalts: A new perspective from petrology, geochemistry, and mineral physics considerations. Journal of Geophysical Research: Solid Earth 2003, 108. doi: 10.1029/2002jb002048.

[47] Zou, H.; Reid, M. R.; Liu, Y.; Yao, Y.; Xu, X.; Fan, Q. Constraints on the origin of historic potassic basalts from northeast China by U-Th disequilibrium data. Chemical Geology 2003, 200, 189-201. doi: 10.1016/S0009-2541(03)00188-8.

[48] Niu, Y.; Collerson, K. D.; Batiza, R.; Wendt, J. I.; Regelous, M Origin of enriched-type mid-ocean ridge basalt at ridges far from mantle plumes: The East Pacific Rise at $11 \circ 20^{\prime} \mathrm{N}$. Journal of Geophysical Research: Solid Earth 1999, 104, 7067-7087. doi: 10.1029/1998JB900037.

[49] Klein, E. M.; Langmuir, C. H. Global correlations of ocean ridge basalt chemistry with axial depth and crustal thickness. Journal of Geophysical Research 1987, 92, 8089. doi: 10.1029/JB092iB08p08089.

[50] Schilling, J. g.; Kingsley, R. H.; Hanan, B. B.; McCully, B. L. $\mathrm{Nd}-\mathrm{Sr}-\mathrm{Pb}$ isotopic variations along the Gulf of Aden: Evidence for Afar Mantle Plume-Continental Lithosphere Interaction. Journal of Geophysical Research 1992, 97, 10927. doi: 10.1029/92JB00415.

[51] Putirka, K. D. Mantle potential temperatures at Hawaii, Iceland, and the mid-ocean ridge system, as inferred from olivine phenocrysts: Evidence for thermally driven mantle plumes. Geochemistry, Geophysics, Geosystems 2005, 6, 1-14. doi: 10.1029/2005GC000915.

[52] Herzberg, C.; Asimow, P. D. PRIMELT3 MEGA. XLSM software for primary magma calculation: Peridotite primary magma $\mathrm{MgO}$ contents from the liquidus to the solidus. Geochemistry, Geophysics, Geosystems 2015, 16, 563-578. doi: 10.1002/2014GC005631.

[53] Putirka, K. D.; Perfit, M.; Ryerson, F. J.; Jackson, M. G. Ambient and excess mantle temperatures, olivine thermometry, and active vs. passive upwelling. Chemical Geology 2007, 241, 177-206. doi: 10.1016/j.chemgeo.2007.01.014.
[54] Albarede, F. How deep do common basaltic magmas form and differentiate? Journal of Geophysical Research, 1992, 97. doi: 10.1029/91jb02927.

[55] Haase, K. M. The relationship between the age of the lithosphere and the composition of oceanic magmas: Constraints on partial melting, mantle sources and the thermal structure of the plates. Earth and Planetary Science Letters 1996, 144, 75-92. doi: 10.1016/0012-821x(96)00145-8.

[56] Campbell, I. H. Testing the plume theory. Chemical Geology, 2007, 241, 153-176. doi: 10.1016/j.chemgeo.2007.01.024.

[57] Hole, M. J.; Millett, J. M. Controls of Mantle Potential Temperature and Lithospheric Thickness on Magmatism in the North Atlantic Igneous Province. Journal of Petrology 2016, 57, 417-436. doi: 10.1093/petrology/egw014.

[58] Tapponnier, P.; Peltzer, G.; Armijo, R. On the mechanics of the collision between India and Asia. Geological Society, London, Special Publications 1986, 19, 113-157. doi: 10.1144/GSL.SP.1986.019.01.07.

[59] Flower, M.; Tamaki, K.; Hoang, N. Mantle extrusion: A model for dispersed volcanism and DUPAL-like asthenosphere in East Asia and the western Pacific. Mantle Dynamics and Plate Interactions in East Asia 1998, 27, 67-88. doi: 10.1029/GD027p0067.

[60] Zhang, Y.; Yu, K.; Qian, H. LA-ICP-MS analysis of clinopyroxenes in basaltic pyroclastic rocks from the xisha islands, Northwestern South China sea. Minerals 2018, 8, 7-15. doi: $10.3390 / \min 8120575$.

[61] Zhang, W. Characteristic of continental rift volcanic activities in Sanshui Basin and early evolution of South China SEA. PhD thesis, China University of Geosciences (Beijing), 2013.

[62] Xiao, L.; Zhou, H.; Dong, Y.; Zeng, G.; Wang, F. Geochemistry and petrogenesis of Cenozoic volcanic rocks from Sanshui Basin: Implications for spatial and temporal variation of rock types and on the formation of South China Sea constraintsS. Geotectonica et Metallogenia 2006, 30, 72-81.

[63] Dong, Y.; Xiao, L.; Zhou, H.; Zeng, G.; Wang, F.; Wang, X.; Xiang, H.; Zhao, T.; Liu, X. Spatial distribution and etrological characteristics of the bimodal volcanic rocks from Sanshui basin, Guangdong province: Implication for basin dynamics. Geotectonica et Metallogenia 2006, 30, 82-92.

[64] Tapponnier, P.; Peltzer, G.; Le Dain, A. Y.; Armijo, R.; Cobbold, P. Propagating extrusion tectonics in Asia: new insights from simple experiments with plasticine. Geology 1982, 10, 611-616. doi: 10.1130/0091-7613(1982)10<611:PETIAN>2.0. CO; 2.

[65] Yao, B.; Wan, L. Variation of the lithospheric thickness in the South China Sea and its tectonic signification. Geology in China 2010, 37.

[66] Ren, J. Y.; Lei, C.; Wang, S.; Li, T.; Zhang, X. L. Tectonic Stratigraphic Framework of the Yinggehai-Qiongdongnan Basins and Its Implication for Tectonics Province Division in South China Sea. Chinese Journal of Geophysics 2013, 54, 1124-1137. doi: 10.1002/cjg2.1689. 